Complete list of Darboux integrable chains of the form $t_{1 x}=t_{X}+d\left(t, t_{1}\right)$

Ismagil Habibullin, Natalya Zheltukhina, and Aslı Pekcan

Citation: Journal of Mathematical Physics 50, 102710 (2009);

View online: https://doi.org/10.1063/1.3251334

View Table of Contents: http://aip.scitation.org/toc/jmp/50/10

Published by the American Institute of Physics

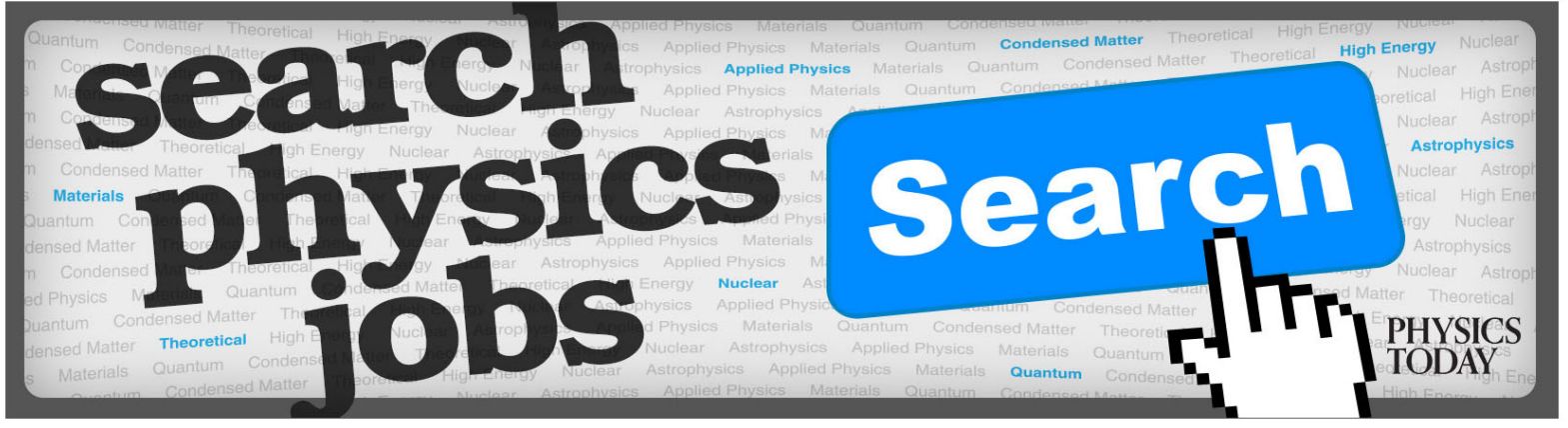




\title{
Complete list of Darboux integrable chains of the form $t_{1 x}=t_{x}+d\left(t, t_{1}\right)$
}

\author{
Ismagil Habibullin, ${ }^{1, a)}$ Natalya Zheltukhina, ${ }^{2, b)}$ and Aslı Pekcan ${ }^{2}$ \\ ${ }^{1}$ Ufa Institute of Mathematics, Russian Academy of Science, Chernyshevskii Str., \\ 112, Ufa 450077, Russia \\ ${ }^{2}$ Department of Mathematics, Faculty of Science, Bilkent University, \\ 06800 Ankara, Turkey
}

(Received 28 July 2009; accepted 23 September 2009; published online 30 October 2009)

We study differential-difference equation $\quad(d / d x) t(n+1, x)=f(t(n, x), t(n$ $+1, x),(d / d x) t(n, x))$ with unknown $t(n, x)$ depending on continuous and discrete variables $x$ and $n$. Equation of such kind is called Darboux integrable, if there exist two functions $F$ and $I$ of a finite number of arguments $x,\{t(n+k, x)\}_{k=-\infty}^{\infty}$, $\left\{\left(d^{k} / d x^{k}\right) t(n, x)\right\}_{k=1}^{\infty}$, such that $D_{x} F=0$ and $D I=I$, where $D_{x}$ is the operator of total differentiation with respect to $x$ and $D$ is the shift operator: $D p(n)=p(n+1)$. Reformulation of Darboux integrability in terms of finiteness of two characteristic Lie algebras gives an effective tool for classification of integrable equations. The complete list of Darboux integrable equations is given in the case when the function $f$ is of the special form $f(u, v, w)=w+g(u, v)$. (c) 2009 American Institute of Physics. [doi:10.1063/1.3251334]

\section{INTRODUCTION}

In this paper we continue investigation of integrable semidiscrete chains of the form

$$
\frac{d}{d x} t(n+1, x)=f\left(t(n, x), t(n+1, x), \frac{d}{d x} t(n, x)\right),
$$

started in our previous paper ${ }^{1}$ (see also Refs. 2-4). Here $t=t(n, x)$ and $t_{1}=t(n+1, x)$ are unknown. Function $f=f\left(t, t_{1}, t_{x}\right)$ is assumed to be locally analytic and $\partial f / \partial t_{x}$ is not identically zero. Nowadays discrete phenomena are very popular due to their applications in physics, geometry, biology, etc. (see Refs. 5-8 and references therein).

Below we use subindex to indicate the shift of the discrete argument: $t_{k}=t(n+k, x), k \in \mathbb{Z}$, and derivatives with respect to $x: t_{[1]}=t_{x}=(d / d x) t(n, x), t_{[2]}=t_{x x}=\left(d^{2} / d x^{2}\right) t(n, x), t_{[m]}=\left(d^{m} / d x^{m}\right) t(n, x)$, $m \in \mathbb{N}$. Introduce the set of dynamical variables containing $\left\{t_{k}\right\}_{k=-\infty}^{\infty} ;\left\{t_{[m]}\right\}_{m=1}^{\infty}$.

We denote through $D$ and $D_{x}$ the shift operator and the operator of the total derivative with respect to $x$ correspondingly. For instance, $D h(n, x)=h(n+1, x)$ and $D_{x} h(n, x)=(d / d x) h(n, x)$.

Functions $I$ and $F$, both depending on $x$ and a finite number of dynamical variables, are called, respectively, $n$ - and $x$-integrals of (1), if $D I=I$ and $D_{x} F=0$ (see also Ref. 9). One can see that any $n$-integral $I$ does not depend on variables $t_{m}, m \in \mathbb{Z} \backslash\{0\}$, and any $x$-integral $F$ does not depend on variables $t_{[m]}, m \in \mathbb{N}$.

Chain (1) is called Darboux integrable if it admits a nontrivial $n$-integral and a nontrivial $x$-integral.

Note that all Darboux integrable chains of the form (1) are reduced to the d'Alembert equation $w_{1 x}-w_{x}=0$ by the following "differential" substitution $w=F+I$. Indeed, $D_{x}(D-1) w=(D-1) D_{x} F$ $+D_{x}(D-1) I=0$. This implies that two arbitrary Darboux integrable chains of the form (1)

\footnotetext{
${ }^{a)}$ Electronic mail: habibullinismagil@gmail.com.

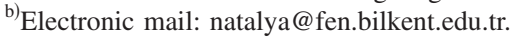




$$
u_{1 x}=f\left(u, u_{1}, u_{x}\right), \quad v_{1 x}=\tilde{f}\left(v, v_{1}, v_{x}\right)
$$

are connected with one another by the substitution

$$
F\left(x, u, u_{1}, u_{-1}, \ldots\right)+I\left(x, u, u_{x}, u_{x x}, \ldots\right)=\widetilde{F}\left(x, v, v_{1}, v_{-1}, \ldots\right)+\widetilde{I}\left(x, v, v_{x}, v_{x x}, \ldots\right),
$$

which is evidently split down into two relations,

$$
F\left(x, u, u_{1}, u_{-1}, \ldots\right)=\widetilde{F}\left(x, v, v_{1}, v_{-1}, \ldots\right)-h, \quad I\left(x, u, u_{x}, u_{x x}, \ldots\right)=\widetilde{I}\left(x, v, v_{x}, v_{x x}, \ldots\right)+h,
$$

where $h$ is some constant.

The idea of such kind integrability goes back to Laplace's discovery of cascade method of integration of linear hyperbolic-type partial differential equation with variable coefficients made in 1773 (see Ref. 10). Roughly speaking the Laplace theorem claims that a linear hyperbolic partial differential equation admits general solution in a closed form if and only if its sequence of Laplace invariants terminates at both ends (see Ref. 11). More than hundred years later Darboux applied the cascade method to the nonlinear case. He proved that a nonlinear hyperbolic equation is integrable (Darboux integrable) if and only if the Laplace sequence of the linearized equation terminates at both ends. This result has been rediscovered very recently by Anderson and Kamran ${ }^{12}$ and Sokolov and Zhiber. ${ }^{13}$

An alternative approach was suggested by Shabat and Yamilov in 1981 (see Ref. 14). They assigned two Lie algebras, called characteristic Lie algebras, to each hyperbolic equation and proved that the equation is Darboux integrable if and only if both characteristic Lie algebras are of finite dimensions.

The purpose of the present article is to study characteristic Lie algebras of the chain (1) introduced in our papers ${ }^{2-4}$ and convince the reader that in the discrete case these algebras provide a very effective classification tool.

We denote through $L_{x}$ and $L_{n}$ characteristic Lie algebras in $x$ - and $n$-directions, respectively. Remind the definition of $L_{x}$. Rewrite first the chain (1) in the inverse form $t_{x}(n-1, x)$ $=g\left(t(n, x), t(n-1, x), t_{x}(n, x)\right)$. It can be done (at least locally) due to the requirement $\left(\partial f / \partial t_{x}\right)$ $\left(t, t_{1}, t_{x}\right) \neq 0$. An $x$-integral $F=F\left(x, t, t_{ \pm 1}, t_{ \pm 2}, \ldots\right)$ solves the equation $D_{x} F=0$. Applying the chain rule, one gets $K F=0$, where

$$
K=\frac{\partial}{\partial x}+t_{x} \frac{\partial}{\partial t}+f \frac{\partial}{\partial t_{1}}+g \frac{\partial}{\partial t_{-1}}+f_{1} \frac{\partial}{\partial t_{2}}+g_{-1} \frac{\partial}{\partial t_{-2}}+\cdots .
$$

Since $F$ does not depend on the variable $t_{x}$, then $X F=0$, where $X=\partial / \partial t_{x}$. Therefore, any vector field from the Lie algebra generated by $K$ and $X$ annulates $F$. This algebra is called the characteristic Lie algebra $L_{x}$ of chain (1) in $x$-direction. The notion of characteristic algebra is very important. One can prove that chain (1) admits a nontrivial $x$-integral if and only if its Lie algebra $L_{x}$ is of finite dimension. The proof of the next classification theorem from Ref. 1 is based on the finiteness of the Lie algebra $L_{x}$.

Theorem 1.1: Chain

$$
t_{1 x}=t_{x}+d\left(t, t_{1}\right)
$$

admits a nontrivial $x$-integral if and only if $d\left(t, t_{1}\right)$ is one of the following kinds:

(1) $d\left(t, t_{1}\right)=A\left(t_{1}-t\right)$,

(2) $d\left(t, t_{1}\right)=c_{1}\left(t_{1}-t\right) t+c_{2}\left(t_{1}-t\right)^{2}+c_{3}\left(t_{1}-t\right)$,

(3) $d\left(t, t_{1}\right)=A\left(t_{1}-t\right) e^{\alpha t}$,

(4) $d\left(t, t_{1}\right)=c_{4}\left(e^{\alpha t_{1}}-e^{\alpha t}\right)+c_{5}\left(e^{-\alpha t_{1}}-e^{-\alpha t}\right)$, where $A=A\left(t_{1}-t\right)$ is an arbitrary function of one variable and $\alpha \neq 0, c_{1} \neq 0, c_{2}, c_{3}, c_{4} \neq 0$, $c_{5} \neq 0$ are arbitrary constants. Moreover, some nontrivial $x$-integrals in each of the cases are

$$
F=x+\int^{t_{1}-t}(d u / A(u)) \text {, if } A(u) \neq 0, F=t_{1}-t \text {, if } A(u) \equiv 0 \text {, }
$$


(ii)

$$
F=\frac{1}{\left(-c_{2}+c_{1}\right)} \ln \left|\left(-c_{2}+c_{1}\right) \frac{t_{2}-t_{1}}{t_{3}-t_{2}}+c_{2}\right|+\frac{1}{c_{2}} \ln \left|c_{2} \frac{t_{2}-t_{1}}{t_{1}-t}-c_{2}+c_{1}\right|
$$

for $c_{2}\left(c_{2}-c_{1}\right) \neq 0$,

$$
F=\ln \left|\frac{t_{2}-t_{1}}{t_{3}-t_{2}}\right|+\frac{t_{2}-t_{1}}{t_{1}-t}
$$

for $c_{2}=0$,

$$
F=\frac{t_{2}-t_{1}}{t_{3}-t_{2}}+\ln \left|\frac{t_{2}-t_{1}}{t_{1}-t}\right|
$$

(iii)

for $c_{2}=c_{1}$.

$$
F=\int^{t_{1}-t} \frac{e^{-\alpha u} d u}{A(u)}-\int^{t_{2}-t_{1}} \frac{d u}{A(u)} .
$$

(iv)

$$
F=\frac{\left(e^{\alpha t}-e^{\alpha t_{2}}\right)\left(e^{\alpha t_{1}}-e^{\alpha t_{3}}\right)}{\left(e^{\alpha t}-e^{\alpha t_{3}}\right)\left(e^{\alpha t_{1}}-e^{\alpha t_{2}}\right)} .
$$

In what follows we study semidiscrete chains (3) admitting not only nontrivial $x$-integrals but also nontrivial $n$-integrals. First of all we will give an equivalent algebraic formulation of the $n$-integral existence problem. Rewrite the equation $D I=I$ defining $n$-integral in an enlarged form,

$$
I\left(x, t_{1}, f, f_{x}, \ldots\right)=I\left(x, t, t_{x}, t_{x x}, \ldots\right) .
$$

The left hand side contains the variable $t_{1}$ while the right hand side does not. Hence we have $D^{-1}\left(d / d t_{1}\right) D I=0$, i.e., the $n$-integral is in the kernel of the operator

$$
Y_{1}=D^{-1} Y_{0} D
$$

where

$$
Y_{1}=\frac{\partial}{\partial t}+D^{-1}\left(Y_{0} f\right) \frac{\partial}{\partial t_{x}}+D^{-1} Y_{0}\left(f_{x}\right) \frac{\partial}{\partial t_{x x}}+D^{-1} Y_{0}\left(f_{x x}\right) \frac{\partial}{\partial t_{x x x}}+\cdots
$$

and

$$
Y_{0}=\frac{d}{d t_{1}} .
$$

It can easily be shown that for any natural $j$ the equation $D^{-j} Y_{0} D^{j} I=0$ holds. Direct calculations show that

$$
D^{-j} Y_{0} D^{j}=X_{j-1}+Y_{j}, \quad j \geq 2,
$$

where

$$
Y_{j+1}=D^{-1}\left(Y_{j} f\right) \frac{\partial}{\partial t_{x}}+D^{-1} Y_{j}\left(f_{x}\right) \frac{\partial}{\partial t_{x x}}+D^{-1} Y_{j}\left(f_{x x}\right) \frac{\partial}{\partial t_{x x x}}+\cdots, \quad j \geq 1,
$$




$$
X_{j}=\frac{\partial}{\partial_{t_{-j}}}, \quad j \geq 1 .
$$

The following theorem defines the characteristic Lie algebra $L_{n}$ of the chain (1).

Theorem 1.2: (Ref. 3) Equation (1) admits a nontrivial n-integral if and only if the following two conditions hold.

(1) Linear space spanned by the operators $\left\{Y_{j}\right\}_{1}^{\infty}$ is of finite dimension, denote this dimension by $N$.

(2) Lie algebra $L_{n}$ generated by the operators $Y_{1}, Y_{2}, \ldots, Y_{N}, X_{1}, X_{2}, \ldots, X_{N}$ is of finite dimension. We call $L_{n}$ the characteristic Lie algebra of (1) in the direction of $n$.

We use $x$-integral classification Theorem 1.1 and $n$-integral existence Theorem 1.2 to obtain the complete list of Darboux integrable chains of the form (3). The statement of this main result of the present paper is given in the next theorem.

Theorem 1.3: Chain (3) admits nontrivial $x$ - and n-integrals if and only if $d\left(t, t_{1}\right)$ is one of the kind.

(1) $d\left(t, t_{1}\right)=A\left(t_{1}-t\right)$, where $A\left(t_{1}-t\right)$ is given in implicit form $A\left(t_{1}-t\right)=(d / d \theta) P(\theta), t_{1}-t=P(\theta)$, with $P(\theta)$ being an arbitrary quasipolynomial, i.e., a function satisfying an ordinary differential equation,

$$
P^{(N+1)}=\mu_{N} P^{(N)}+\cdots+\mu_{1} P^{\prime}+\mu_{0} P,
$$

with constant coefficients $\mu_{k}, 0 \leq k \leq N$.

(2) $d\left(t, t_{1}\right)=C_{1}\left(t_{1}^{2}-t^{2}\right)+C_{2}\left(t_{1}-t\right)$.

(3) $d\left(t, t_{1}\right)=\sqrt{C_{3} e^{2 \alpha t_{1}}+C_{4} e^{\alpha\left(t_{1}+t\right)}+C_{3} e^{2 \alpha t}}$.

(4) $d\left(t, t_{1}\right)=C_{5}\left(e^{\alpha t_{1}}-e^{\alpha t}\right)+C_{6}\left(e^{-\alpha t_{1}}-e^{-\alpha t}\right)$,

where $\alpha \neq 0, C_{i}, 1 \leq i \leq 6$, are arbitrary constants. Moreover, some nontrivial $x$-integrals $F$ and $n$-integrals $I$ in each of the cases are the following.

(i) $\quad F=x-\int^{t_{1}-t} d s / A(s), I=L\left(D_{x}\right) t_{x}$, where $L\left(D_{x}\right)$ is a differential operator which annihilates $(d / d \theta) P(\theta)$ where $D_{x} \theta=1$.

(ii) $F=\left\{\left(t_{3}-t_{1}\right)\left(t_{2}-t\right)\right\} /\left\{\left(t_{3}-t_{2}\right)\left(t_{1}-t\right)\right\}, I=t_{x}-C_{1} t^{2}-C_{2} t$.

(iii) $F=\int^{t_{1}-t} e^{-\alpha s} d s / \sqrt{C_{3} e^{2 \alpha s}+C_{4} e^{\alpha s}+C_{3}}-\int^{t_{2}-t_{1}} d s / \sqrt{C_{3} e^{2 \alpha s}+C_{4} e^{\alpha s}+C_{3}}, I=2 t_{x x}-\alpha t_{x}^{2}-\alpha C_{3} e^{2 \alpha t}$.

(iv) $F=\left\{\left(e^{\alpha t}-e^{\alpha t_{2}}\right)\left(e^{\alpha t_{1}}-e^{\alpha t_{3}}\right)\right\} /\left\{\left(e^{\alpha t}-e^{\alpha t_{3}}\right)\left(e^{\alpha t_{1}}-e^{\alpha t_{2}}\right)\right\}, I=t_{x}-C_{5} e^{\alpha t}-C_{6} e^{-\alpha t}$.

Equation of the form $\tau_{x}=A(\tau)$, where $\tau=t_{1}-t$, is integrated in quadratures. But to get the final answer one should evaluate the integral and then find the inverse function. The general solution is given in an explicit form,

$$
t(n, x)=t(0, x)+\sum_{j=0}^{n-1} P\left(x+c_{j}\right)
$$

where $t(0, x)$ and $c_{j}$ are arbitrary functions of $x$ and $j$, respectively, and $A(\tau)=P^{\prime}(\theta), t_{1}-t=P(\theta)$. Actually we have $\tau_{x}=P_{\theta}(\theta) \theta_{x}=P_{\theta}(\theta)$, which implies $\theta_{x}=1$, so that $\tau(n, x)=P\left(x+c_{n}\right)$. By solving the equation $t(n+1, x)-t(n, x)=P\left(x+c_{n}\right)$ one gets the answer above. Requirement for $\tau_{x}=A(\tau)$ to be Darboux integrable induces condition on function $P$ to satisfy a linear ordinary differential equation with constant coefficients.

The $x$-integrals in the cases (2) and (4) given in Theorem 1.3 are written as cross ratios of four points $t, t_{1}, t_{2}, t_{3}$ and, respectively, points $e^{t}, e^{t_{1}}, e^{t_{2}}, e^{t_{3}}$. Due to the well known theorem, given four points $z_{1}, z_{2}, z_{3}, z_{4}$ in the projective complex plane CP can be mapped to other given four points $w_{1}, w_{2}, w_{3}, w_{4}$ by one and the same Möbius transformation, 


$$
z=R(w):=\frac{a_{11} w+a_{12}}{a_{21} w+a_{22}},
$$

such that $z_{j}=R\left(w_{j}\right)$, where $j=1,2,3,4$, if and only if

$$
\frac{z_{4}-z_{2}}{z_{4}-z_{3}} \frac{z_{3}-z_{1}}{z_{2}-z_{1}}=\frac{w_{4}-w_{2}}{w_{4}-w_{3}} \frac{w_{3}-w_{1}}{w_{2}-w_{1}}
$$

Evidently function $F$ for the case (2) [as well as for the case (4)] can immediately be found from the equation $I=c(x)$ which is equivalent to Riccati equation $t_{x}=C_{1} t^{2}+C_{2} t+c(x)$. It is well known that cross ratio of four different solutions of the Riccati equation does not depend on $x$.

Studying the examples below we briefly discuss connection between discrete models and their continuum analogs. The case (3) with $C_{3}=1$ and $\alpha=1$ leads in the continuum limit to the equation

$$
u_{x y}=e^{u} \sqrt{u_{y}^{2}+1}
$$

found earlier in Ref. 13. Indeed set $t(n, x)=u(y, x)$ and $C_{4}=-2+\epsilon^{2}$, where $y=n \epsilon$. Then substitute $\tau=\epsilon u_{y}+O\left(\epsilon^{2}\right)$ as $\epsilon \rightarrow 0$ into the equation $t_{1 x}-t_{x}=e^{t} \sqrt{e^{2 \tau}+C_{4} e^{\tau}+1}$ and evaluate the limit as $\epsilon \rightarrow 0$ to get (12). It is remarkable that Eq. (12) has the same integral (y-integral) $I=2 u_{x x}-u_{x}^{2}-e^{2 u}$ as its discrete counterpart.

The chain $t_{1 x}-t_{x}=\left(e^{t_{1}}-e^{t}\right) / 2$ goes to the equation $u_{x y}=\frac{1}{2} e^{u} u_{y}$ in the continuum limit. Its $n$-integral $I=t_{x}-\frac{1}{2} e^{t}$ coincides with the corresponding $y$-integral of the continuum analog. The Darboux integrable chain $t_{1 x}-t_{x}=C e^{\left(t_{1}+t\right) / 2}$ [it comes from the case (3) for appropriate choice of the parameters] being a discrete version of the Liouville equation $u_{x y}=e^{u}$, also has a common integral $I=2 t_{x x}-t_{x}^{2}$ with its continuum limit equation. Note that the chain defines the Bäcklund transform for the Liouville equation.

Let us comment the list of the equations in Theorem 1.3. Case (1) is degenerate, it is reduced to a first order ordinary differential equation and easily integrated. Equation (2) with $C_{2}=0$ is given in Ref. 9. Case (3) for $C_{4}= \pm C_{3}$ is found in Ref. 15. To the best of our knowledge Eqs. (2)-(4) are new except these two cases.

The article is organized as follows. In Sec. II general results related to the Lie algebra $L_{n}$ of Eq. (1) are given. Section III is split into four subsections. Theorem 1.1 from Sec. I gives a complete list of Eq. (3) admitting nontrivial $x$-integral. This list consists of four different types of equations (3). In each subsection of Sec. III one of these four different types from Theorem 1.1 is treated by imposing additional condition for an equation to possess nontrivial $n$-integrals. The conclusion is provided in Sec. IV.

\section{GENERAL RESULTS}

Define a class $\mathbf{F}$ of locally analytic functions each of which depends only on a finite number of dynamical variables. In particular, we assume that $f\left(t, t_{1}, t_{x}\right) \in \mathbf{F}$. We will consider vector fields given as infinite formal series of the form

$$
Y=\sum_{k=0}^{\infty} y_{k} \frac{\partial}{\partial t_{[k]}}
$$

with coefficients $y_{k} \in \mathbf{F}$. Introduce notions of linearly dependent and independent sets of the vector fields (13). Denote through $P_{N}$ the projection operator acting according to the rule

$$
P_{N}(Y)=\sum_{k=0}^{N} y_{k} \frac{\partial}{\partial t_{[k]}} .
$$

First we consider finite vector fields as 


$$
Z=\sum_{k=0}^{N} z_{k} \frac{\partial}{\partial t_{[k]}} .
$$

We say that a set of finite vector fields $Z_{1}, Z_{2}, \ldots, Z_{m}$ is linearly dependent in some open region $\mathbf{U}$, if there is a set of functions $\lambda_{1}, \lambda_{2}, \ldots, \lambda_{m} \in \mathbf{F}$ defined on $\mathbf{U}$ such that the function $\left|\lambda_{1}\right|^{2}+\left|\lambda_{2}\right|^{2}$ $+\cdots+\left|\lambda_{m}\right|^{2}$ does not vanish identically and the condition

$$
\lambda_{1} Z_{1}+\lambda_{2} Z_{2}+\cdots+\lambda_{m} Z_{m}=0
$$

holds for each point of region $\mathbf{U}$.

We call a set of the vector fields $Z_{1}, Z_{2}, \ldots, Z_{m}$ of the form (13) linearly dependent in the region $\mathbf{U}$ if for each natural $N$ the following set of finite vector fields $P_{N}\left(Z_{1}\right), P_{N}\left(Z_{2}\right), \ldots, P_{N}\left(Z_{m}\right)$ is linearly dependent in this region. Otherwise we call the set $Z_{1}, Z_{2}, \ldots, Z_{m}$ linearly independent in $\mathbf{U}$.

Now we give some properties of the characteristic Lie algebra introduced in Theorem 1.2. The proof of the first two lemmas can be found in Ref. 3. However, for the reader's convenience we still give the proof of the second lemma.

Lemma 2.1: If for some integer $N$ the operator $Y_{N+1}$ is a linear combination of the operators $Y_{i}$ with $i \leq N$ : $Y_{N+1}=\alpha_{1} Y_{1}+\alpha_{2} Y_{2}+\ldots+\alpha_{N} Y_{N}$, then for any integer $j>N$, we have a similar expression $Y_{j}=\beta_{1} Y_{1}+\beta_{2} Y_{2}+\cdots+\beta_{N} Y_{N}$.

Lemma 2.2: The following commutativity relations take place: $\left[Y_{0}, X_{1}\right]=0,\left[Y_{0}, Y_{1}\right]=0$, and $\left[X_{1}, D X_{1} D^{-1}\right]=0$.

Proof: We have

$$
\begin{gathered}
{\left[Y_{0}, X_{1}\right]=\left[\frac{d}{d t_{1}}, \frac{d}{d t_{-1}}\right]=0,} \\
{\left[Y_{0}, Y_{1}\right]=D^{-1}\left[D Y_{0} D^{-1}, Y_{0}\right] D=D^{-1}\left[\frac{d}{d t_{2}}, \frac{d}{d t_{1}}\right] D=0,} \\
{\left[X_{1}, D X_{1} D^{-1}\right]=D\left[D^{-1} X_{1} D, X_{1}\right] D^{-1}=D\left[X_{2}, X_{1}\right] D^{-1}=0 .}
\end{gathered}
$$

Note that

$$
Y_{k+1}=D^{-1} Y_{k} D, \quad k \geq 2, \quad D^{-1} Y_{1} D=X_{1}+Y_{2} .
$$

The next three statements turned out to be very useful for studying the characteristic Lie algebra $L_{n}$.

Lemma 2.3: (Reference 1) If the Lie algebra generated by the vector fields $S_{0}=\sum_{j=-\infty}^{\infty} \partial / \partial w_{j}$ and $S_{1}=\Sigma_{j=-\infty}^{\infty} c\left(w_{j}\right) \partial / \partial w_{j}$ is of finite dimension then $c(w)$ is one of the forms

(1) $c(w)=a_{1}+a_{2} e^{\lambda w}+a_{3} e^{-\lambda w}$,

(2) $c(w)=a_{1}+a_{2} w+a_{3} w^{2}$, where $\lambda \neq 0, a_{1}, a_{2}$, and $a_{3}$ are some constants.

Lemma 2.4:

(1) Suppose that the vector field

$$
Y=\alpha(0) \frac{\partial}{\partial t}+\alpha(1) \frac{\partial}{\partial t_{x}}+\alpha(2) \frac{\partial}{\partial t_{x x}}+\cdots,
$$

where $\alpha_{x}(0)=0$ solves the equation $\left[D_{x}, Y\right]=\sum_{k=-\infty, k \neq 0}^{\infty} \beta(k) \partial / \partial t_{k}$, then $Y=\alpha(0) \partial / \partial t$.

(2) Suppose that the vector field 


$$
Y=\alpha(1) \frac{\partial}{\partial t_{x}}+\alpha(2) \frac{\partial}{\partial t_{x x}}+\alpha(3) \frac{\partial}{\partial t_{x x x}}+\cdots
$$

solves the equation $\left[D_{x}, Y\right]=h Y+\sum_{k=-\infty, k \neq 0}^{\infty} \beta(k) \partial / \partial t_{k}$, where $h$ is a function of variables $t, t_{x}$, $t_{x x}, \ldots, t_{ \pm 1}, t_{ \pm 2}, \ldots$, then $Y=0$.

Lemma 2.5: For any $m \geq 0$, we have

$$
\left[D_{x}, Y_{m}\right]=-\sum_{j=1}^{m} D^{-j}\left(Y_{m-j}(f)\right) Y_{j}-\sum_{k=1}^{\infty} Y_{m}\left(D^{-(k-1)} g\right) \frac{\partial}{\partial t_{-k}}-\sum_{k=1}^{\infty} Y_{m}\left(D^{k-1} f\right) \frac{\partial}{\partial t_{k}} .
$$

In particular,

$$
\begin{gathered}
{\left[D_{x}, Y_{0}\right]=-\sum_{k=1}^{\infty} Y_{0}\left(D^{k-1} f\right) \frac{\partial}{\partial t_{k}},} \\
{\left[D_{x}, Y_{1}\right]=-D^{-1}\left(Y_{0}(f)\right) Y_{1}-\sum_{k=1}^{\infty} Y_{1}\left(D^{-(k-1)} g\right) \frac{\partial}{\partial t_{-k}}-\sum_{k=1}^{\infty} Y_{1}\left(D^{k-1} f\right) \frac{\partial}{\partial t_{k}} .}
\end{gathered}
$$

Both Lemmas 2.4 and 2.5 easily can be derived from the following formula

$$
\left[D_{x}, Y\right]=\left(\alpha_{x}(0)-\alpha(1)\right) \frac{\partial}{\partial t}-\sum_{k=1}^{\infty} Y\left(D^{-(k-1)} g\right) \frac{\partial}{\partial t_{-k}}-\sum_{k=1}^{\infty} Y\left(D^{k-1} f\right) \frac{\partial}{\partial t_{k}}+\sum_{k=1}^{\infty}\left(\alpha_{x}(k)-\alpha(k+1)\right) \frac{\partial}{\partial t_{[k]}}
$$

Suppose that Eq. (1) admits a nontrivial $n$-integral. Then, by Theorem 1.2, its characteristic Lie algebra $L_{n}$ is of finite dimension. Linear space of the basic vector fields $\left\{Y_{k}\right\}_{1}^{\infty}$ is also finite dimensional. We have the following theorem.

Theorem 2.6: Dimension of $\operatorname{span}\left\{Y_{k}\right\}_{1}^{\infty}$ is finite and equal, say $N$ if and only if the following system of equations is consistent:

$$
\begin{gathered}
D_{x}\left(\lambda_{N}\right)=\lambda_{N}\left(A_{N, N}-A_{N+1, N+1}\right)-A_{N+1, N}, \\
D_{x}\left(\lambda_{N-1}\right)=\lambda_{N-1}\left(A_{N-1, N-1}-A_{N+1, N+1}\right)+\lambda_{N} A_{N, N-1}-A_{N+1, N-1}, \\
D_{x}\left(\lambda_{N-2}\right)=\lambda_{N-2}\left(A_{N-2, N-2}-A_{N+1, N+1}\right)+\lambda_{N-1} A_{N-1, N-2}+\lambda_{N} A_{N, N-2}-A_{N+1, N-2}, \\
\vdots \\
D_{x}\left(\lambda_{2}\right)=\lambda_{2}\left(A_{2,2}-A_{N+1, N+1}\right)+\lambda_{3} A_{3,2}+\cdots+\lambda_{N} A_{N, 2}-A_{N+1,2}, \\
D_{x}\left(\lambda_{1}\right)=\lambda_{1}\left(A_{1,1}-A_{N+1, N+1}\right)+\lambda_{2} A_{2,1}+\lambda_{3} A_{3,1}+\cdots+\lambda_{N} A_{N, 1}-A_{N+1,1} . \\
0=\lambda_{1} A_{1,0}+\lambda_{2} A_{2,0}+\lambda_{3} A_{3,0}+\cdots+\lambda_{N} A_{N, 0}-A_{N+1,0} .
\end{gathered}
$$

Here $A_{k, j}=D^{-j}\left(Y_{k-j} f\right)$.

Proof: Suppose that the dimension of $\operatorname{span}\left\{Y_{k}\right\}_{1}^{\infty}$ is finite, say $N$, then, by Lemma 2.1, $Y_{1}, \ldots, Y_{N}$ form a basis in this linear space. So we can find factors $\lambda_{1}, \ldots, \lambda_{N}$ such that 


$$
Y_{N+1}=\lambda_{1} Y_{1}+\lambda_{2} Y_{2}+\cdots+\lambda_{N} Y_{N} .
$$

Take the commutator of both sides with $D_{x}$ and get by using the main commutativity relation (18) the following equation:

$$
\begin{aligned}
-\sum_{j=0}^{N+1} A_{N+1, j} Y_{j}= & D_{x}\left(\lambda_{1}\right) Y_{1}+D_{x}\left(\lambda_{2}\right) Y_{2}+\cdots+D_{x}\left(\lambda_{N}\right) Y_{N} \\
& -\left(\lambda_{1} \sum_{j=0}^{1} A_{1, j} Y_{j}+\lambda_{2} \sum_{j=0}^{2} A_{2, j} Y_{j}+\cdots+\lambda_{N} \sum_{j=0}^{N} A_{N, j} Y_{j}\right) .
\end{aligned}
$$

Now replace $Y_{N+1}$ at the left hand side by (23) and collect coefficients of the independent vector fields to derive the system given in the theorem.

Suppose now that the system (22) in the theorem has a solution. Let us prove that the vector field $Y_{N+1}$ is expressed in the form (23). Let

$$
Z=Y_{N+1}-\lambda_{1} Y_{1}-\lambda_{2} Y_{2}-\cdots-\lambda_{N} Y_{N}
$$

Let us find $\left[D_{x}, Z\right]$.

$$
\begin{aligned}
{\left[D_{x}, Z\right]=} & {\left[D_{x}, Y_{N+1}\right]-D_{x}\left(\lambda_{1}\right) Y_{1}-\cdots-D_{x}\left(\lambda_{N}\right) Y_{N}-\lambda_{1}\left[D_{x}, Y_{1}\right]-\lambda_{2}\left[D_{x}, Y_{2}\right]-\cdots-\lambda_{N}\left[D_{x}, Y_{N}\right] } \\
= & -\sum_{j=0}^{N+1} A_{N+1, j} Y_{j}-D_{x}\left(\lambda_{1}\right) Y_{1}-\cdots-D_{x}\left(\lambda_{N}\right) Y_{N} \\
& -\left(\lambda_{1} \sum_{j=0}^{1} A_{1, j} Y_{j}+\lambda_{2} \sum_{j=0}^{2} A_{2, j} Y_{j}+\cdots+\lambda_{N} \sum_{j=0}^{N} A_{N, j} Y_{j}\right)+\sum_{k=-\infty, k \neq 0}^{\infty} \beta(k) \frac{\partial}{\partial t_{k}} .
\end{aligned}
$$

Replace now $D_{x}\left(\lambda_{1}\right), \ldots, D_{x}\left(\lambda_{N}\right)$ by means of the system (22). After some simplifications one gets

$$
\left[D_{x}, Z\right]=-A_{N+1, N+1} Z+\sum_{k=-\infty, k \neq 0}^{\infty} \beta(k) \frac{\partial}{\partial t_{k}} .
$$

By Lemma 2.4 we get $Z=0$.

The proof of the next three results can be found in Ref. 4 .

Lemma 2.7: If the operator $Y_{2}=0$ then $\left[X_{1}, Y_{1}\right]=0$.

The reverse statement to Lemma 2.7 is not true as the equation $t_{1 x}=t_{x}+e^{t}$ shows (see Lemma 3.4 below).

Lemma 2.8: The operator $Y_{2}=0$ if and only if we have

$$
f_{t}+D^{-1}\left(f_{t_{1}}\right) f_{t_{x}}=0 .
$$

Corollary 2.9: The dimension of the Lie algebra $L_{n}$ associated with $n$-integral is equal to 2 if and only if (26) holds, or the same $Y_{2}=0$.

Now let us introduce vector fields

$$
C_{1}=\left[X_{1}, Y_{1}\right], \quad C_{k}=\left[X_{1}, C_{k-1}\right], \quad k \geq 2 .
$$

It is easy to see that

$$
C_{m}=X_{1}^{m} D^{-1}\left(Y_{0}(f)\right) \frac{\partial}{\partial t_{x}}+X_{1}^{m} D^{-1}\left(Y_{0} D_{x}(f)\right) \frac{\partial}{\partial t_{x x}}+X_{1}^{m} D^{-1}\left(Y_{0} D_{x}^{2}(f)\right) \frac{\partial}{\partial t_{x x x}}+\cdots .
$$

Lemma 2.10: We have 


$$
\left[D_{x}, C_{m}\right]=-g_{t_{x}} X_{1}^{m} D^{-1} Y_{0}(f) X_{1}-X_{1}^{m} D^{-1} Y_{0}(f) Y_{1}-\sum_{j=1}^{m} A_{j}^{(m)} C_{j},
$$

where

$$
A_{j}^{(m)}=X_{1}^{m-j}\left\{C(m, j-1) g_{t_{-1}}-C(m, j) \frac{g_{t}}{g_{t_{x}}}\right\}, \quad m \geq 1, \quad C(m, k)=\frac{m !}{k !(m-k) !} .
$$

In particular,

$$
\left[D_{x}, C_{1}\right]=-g_{t_{x}} X_{1} D^{-1} Y_{0}(f) X_{1}-X_{1} D^{-1} Y_{0}(f) Y_{1}-\left(g_{t_{-1}}-\frac{g_{t}}{g_{t_{x}}}\right) C_{1} .
$$

Proof: We prove the lemma by induction on $m$. Note that for any vector field,

$$
A=\beta(0) \frac{\partial}{\partial t}+\beta(1) \frac{\partial}{\partial t_{x}}+\beta(2) \frac{\partial}{\partial t_{x x}}+\cdots,
$$

acting on the set of functions $H$ depending on variables $t_{-1}, t, t_{[k]}, k \in \mathbb{N}$, formula (21) becomes

$$
\begin{aligned}
{\left[D_{x}, A\right]=} & -\left(\beta(0) g_{t}+\beta(1) g_{t_{x}}\right) \frac{\partial}{\partial t_{-1}}+\left(\beta_{x}(0)-\beta(1)\right) \frac{\partial}{\partial t}+\left(\beta_{x}(1)-\beta(2)\right) \frac{\partial}{\partial t_{x}}+\left(\beta_{x}(2)-\beta(3)\right) \frac{\partial}{\partial t_{x x}} \\
& +\left(\beta_{x}(3)-\beta(4)\right) \frac{\partial}{\partial t_{x x x}}+\cdots .
\end{aligned}
$$

Applying the last formula with $C_{1}$ instead of $A$, we have

$$
\left[D_{x}, C_{1}\right]=-g_{t_{x}} X_{1} D^{-1} Y_{0}(f) X_{1}-X_{1} D^{-1} Y_{0}(f) \frac{\partial}{\partial t}+\sum_{k=1}^{\infty}\left\{D_{x} X_{1} D^{-1} Y_{0} D_{x}^{k-1}(f)-X_{1} D^{-1} Y_{0} D_{x}^{k}(f)\right\} \frac{\partial}{\partial t_{[k]}} .
$$

Since

$$
\left[Y_{0}, D_{x}\right] G\left(t, t_{1}, t_{x}, t_{x x}, t_{x x x}, \ldots\right)=f_{t_{1}} G_{t_{1}}=f_{t_{1}} Y_{0} G, \quad \text { i.e., } \quad Y_{0} D_{x}=D_{x} Y_{0}+f_{t_{1}} Y_{0}
$$

and

$$
\left[D_{x}, X_{1}\right] H\left(t_{-1}, t, t_{x}, t_{x x}, t_{x x x}, \ldots\right)=-g_{t_{-1}} H_{t_{-1}}=-g_{t_{-1}} X_{1} H
$$

then

$$
\begin{aligned}
D_{x} X_{1} D^{-1} Y_{0} D_{x}^{k-1}(f)-X_{1} D^{-1} Y_{0} D_{x}^{k}(f)= & \left\{D_{x} X_{1} D^{-1} Y_{0}-X_{1} D^{-1} Y_{0} D_{x}\right\} D_{x}^{k-1}(f)=\left\{D_{x} X_{1} D^{-1} Y_{0}\right. \\
& \left.-X_{1} D^{-1}\left\{D_{x} Y_{0}+f_{t_{1}} Y_{0}\right\}\right\} D_{x}^{k-1}(f) \\
= & {\left[D_{x}, X_{1}\right] D^{-1} Y_{0} D_{x}^{k-1}(f) X_{1}\left(D^{-1} Y_{0}(f)\right) D^{-1} Y_{0} D_{x}^{k-1}(f) } \\
& -D^{-1}\left(Y_{0}(f)\right) X_{1} D^{-1} Y_{0} D_{x}^{k-1}(f)=-g_{t_{-1}} X_{1} D^{-1} Y_{0} D_{x}^{k-1}(f) \\
& -X_{1} D^{-1}\left(Y_{0}(f)\right) D^{-1} Y_{0} D_{x}^{k-1}(f)-D^{-1}\left(Y_{0}(f)\right) X_{1} D^{-1} Y_{0} D_{x}^{k-1}(f) .
\end{aligned}
$$

Therefore, 


$$
\begin{aligned}
{\left[D_{x}, C_{1}\right]=} & -g_{t_{x}} X_{1} D^{-1} Y_{0}(f) X_{1}-X_{1} D^{-1} Y_{0}(f) \frac{\partial}{\partial t}-\sum_{k=1}^{\infty} X_{1}\left(D^{-1} Y_{0}(f)\right) D^{-1} Y_{0} D_{x}^{k-1}(f) \frac{\partial}{\partial t_{[k]}} \\
& -g_{t_{-1}} \sum_{k=1}^{\infty} X_{1} D^{-1} Y_{0} D_{x}^{k-1}(f) \frac{\partial}{\partial t_{[k]}}-D^{-1}\left(Y_{0}(f)\right) \sum_{k=1}^{\infty} X_{1} D^{-1} Y_{0} D_{x}^{k-1}(f) \frac{\partial}{\partial t_{[k]}}=-g_{t_{x}} X_{1} D^{-1} Y_{0}(f) X_{1} \\
& -X_{1} D^{-1} Y_{0}(f) Y_{1}-\left(g_{t_{-1}}+D^{-1}\left(f_{t_{1}}\right)\right) C_{1}
\end{aligned}
$$

that proves the base of mathematical induction. Assuming Eq. (29) is true for $m-1$, we have

$$
\begin{aligned}
{\left[D_{x}, C_{m}\right]=} & {\left[D_{x},\left[X_{1}, C_{m-1}\right]\right]=-\left[X_{1},\left[C_{m-1}, D_{x}\right]\right]-\left[C_{m-1},\left[D_{x}, X_{1}\right]\right]=\left[X_{1},\left[D_{x}, C_{m-1}\right]\right]+\left[C_{m-1}, g_{t_{-1}} X_{1}\right] } \\
= & {\left[X_{1},\left[D_{x}, C_{m-1}\right]\right]+C_{m-1}\left(g_{t_{-1}}\right) X_{1}-g_{t_{-1}} C_{m}=\left[X_{1},-g_{t_{x}} X_{1}^{m-1} D^{-1} Y_{0}(f) X_{1}-X_{1}^{m-1} D^{-1} Y_{0}(f) Y_{1}\right.} \\
& \left.-\sum_{j=1}^{m-1} A_{j}^{(m-1)} C_{j}\right]+g_{t_{-1} t_{x}} X_{1}^{m-1} D^{-1} Y_{0}(f) X_{1}-g_{t_{-1}} C_{m}=-g_{t_{-1} t_{x}} X_{1}^{m-1} D^{-1} Y_{0}(f) X_{1} \\
& -g_{t_{x}} X_{1}^{m} D^{-1} Y_{0}(f) X_{1}-X_{1}^{m} D^{-1} Y_{0}(f) Y_{1}-X_{1}^{m-1} D^{-1} Y_{0}(f) C_{1}-\sum_{j=1}^{m-1} X_{1}\left(A_{j}^{(m-1)}\right) C_{j} \\
& -\sum_{j=1}^{m-1} A_{j}^{(m-1)} C_{j+1}+g_{t_{-1} t_{x}} X_{1}^{m-1} D^{-1} Y_{0}(f) X_{1}-g_{t_{-1}} C_{m}=-g_{t_{x}} X_{1}^{m} D^{-1} Y_{0}(f) X_{1}-X_{1}^{m} D^{-1} Y_{0}(f) Y_{1} \\
& -\left\{A_{m-1}^{(m-1)}+g_{t_{-1}}\right\} C_{m}-\left\{X_{1}^{m-1} D^{-1} Y_{0}(f)+X_{1}\left(A_{1}^{(m-1)}\right)\right\} C_{1}-\sum_{j=2}^{m-1}\left\{X_{1}\left(A_{j}^{(m-1)}\right)+A_{j-1}^{(m-1)}\right\} C_{j} \\
= & -g_{t_{x}} X_{1}^{m} D^{-1} Y_{0}(f) X_{1}-X_{1}^{m} D^{-1} Y_{0}(f) Y_{1}-\sum_{j=1}^{m} A_{j}^{(m)} C_{j},
\end{aligned}
$$

where

$$
\begin{aligned}
A_{1}^{(m)}= & X_{1}^{m-1} D^{-1} Y_{0}(f)+X_{1}\left(A_{1}^{(m-1)}\right)=X_{1}^{m-1}\left\{-\frac{g_{t}}{g_{t_{x}}}\right\}+X_{1} X_{1}^{m-2}\left\{C(m-1,0) g_{t_{-1}}-C(m-1,1) \frac{g_{t}}{g_{t_{x}}}\right\} \\
= & X_{1}^{m-1}\left\{C(m, 0) g_{t_{-1}}-C(m, 1) \frac{g_{t}}{g_{t_{x}}}\right\}, \\
A_{j}^{(m)}= & X_{1}\left(A_{j}^{(m-1)}\right)+A_{j-1}^{(m-1)}=X_{1} X_{1}^{m-1-j}\left\{C(m-1, j-1) g_{t_{-1}}-C(m-1, j) \frac{g_{t}}{g_{t_{x}}}\right\} \\
& +X_{1}^{m-j}\left\{C(m-1, j-2) g_{t_{-1}}-C(m-1, j-1) \frac{g_{t}}{g_{t_{x}}}\right\}=X_{1}^{m-j}\left\{C(m, j-1) g_{t_{-1}}-C(m, j) \frac{g_{t}}{g_{t_{x}}}\right\}, \\
& A_{m}^{m}=A_{m-1}^{(m-1)}+g_{t_{-1}}=(m-1) g_{t_{-1}}-\frac{g_{t}}{g_{t_{x}}}+g_{t_{-1}}=m g_{t_{-1}}-\frac{g_{t}}{g_{t_{x}}}
\end{aligned}
$$

that finishes the proof of the lemma.

Assume equation $t_{1 x}=f\left(t, t_{1}, t_{x}\right)$ admits a nontrivial $n$-integral. Then we know that the dimension of Lie algebra $L_{n}$ is at least 2 by Corollary 2.9. 
Consider case when the dimension of $L_{n}$ is at least 3 and $C_{1} \neq 0$. Since linear space generated by vector fields $C_{1}, C_{2}, C_{3}, \ldots$, is of finite dimension, then there exists a natural number $N$, such that

$$
C_{N+1}=\mu_{1} C_{1}+\mu_{2} C_{2}+\cdots+\mu_{N} C_{N},
$$

and $C_{1}, C_{2}, \ldots, C_{N}$ are linearly independent. By Lemma 2.10 we have

$$
\begin{aligned}
{\left[D_{x}, C_{N+1}\right]=} & -g_{t_{x}} A_{0}^{(N+1)} X_{1}-A_{0}^{(N+1)} Y_{1}-A_{1}^{(N+1)} C_{1}-\cdots-A_{N}^{(N+1)} C_{n}-A_{N+1}^{(N+1)}\left\{\mu_{1} C_{1}+\mu_{2} C_{2}+\cdots\right. \\
& \left.+\mu_{N} C_{N}\right\},
\end{aligned}
$$

where $A_{0}^{(k)}=X_{1}^{k} D^{-1} Y_{0}(f)$. On the other hand,

$$
\begin{aligned}
{\left[D_{x}, C_{N+1}\right]=} & D_{x}\left(\mu_{1}\right) C_{1}+D_{x}\left(\mu_{2}\right) C_{2}+\cdots+D_{x}\left(\mu_{N}\right) C_{N}+\mu_{1}\left(-g_{t_{x}} A_{0}^{(1)} X_{1}-A_{0}^{(1)} Y_{1}-A_{1}^{(1)} C_{1}\right) \\
& +\mu_{2}\left(-g_{t_{x}} A_{0}^{(2)} X_{1}-A_{0}^{(2)} Y_{1}-A_{1}^{(2)} C_{1}-A_{2}^{(2)} C_{2}\right)+\cdots+\mu_{N}\left(-g_{t_{x}} A_{0}^{(N)} X_{1}-A_{0}^{(N)} Y_{1}\right. \\
& \left.-A_{1}^{(N)} C_{1}-\cdots-A_{N}^{(N)} C_{N}\right) .
\end{aligned}
$$

Linear independence of $X_{1}, Y_{1}, C_{1}, C_{2}, \ldots, C_{N}$ allows us to compare coefficients before $X_{1}, C_{k}$, $1 \leq k \leq N$ in the last two presentations for $\left[D_{x}, C_{N+1}\right]$. We have

$$
\begin{gathered}
-A_{0}^{(N+1)}=-\mu_{1} A_{0}^{(1)}-\mu_{2} A_{0}^{(2)}-\cdots-\mu_{N} A_{0}^{(N)}, \\
-A_{1}^{(N+1)}-\mu_{1} A_{N+1}^{(N+1)}=-\mu_{1} A_{1}^{(1)}-\mu_{2} A_{1}^{(2)}-\cdots-\mu_{N} A_{1}^{(N)}+D_{x}\left(\mu_{1}\right), \\
-A_{k}^{(N+1)}-\mu_{k} A_{N+1}^{(N+1)}=-\left\{\sum_{j=k}^{N} \mu_{j} A_{k}^{(j)}\right\}+D_{x}\left(\mu_{k}\right), \quad 2 \leq k \leq N-3, \\
-A_{N-2}^{(N+1)}-\mu_{N-2} A_{N+1}^{(N+1)}=-\mu_{N-2} A_{N-2}^{(N-2)}-\mu_{N-1} A_{N-2}^{(N-1)}-\mu_{N} A_{N-2}^{(N)}+D_{x}\left(\mu_{N-2}\right), \\
-A_{N-1}^{(N+1)}-\mu_{N-1} A_{N+1}^{(N+1)}=-\mu_{N-1} A_{N-1}^{(N-1)}-\mu_{N} A_{N-1}^{(N)}+D_{x}\left(\mu_{N-1}\right), \\
-A_{N}^{(N+1)}-\mu_{N} A_{N+1}^{(N+1)}=-\mu_{N} A_{N}^{(N)}+D_{x}\left(\mu_{N}\right) .
\end{gathered}
$$

Thus we have proven the following theorem.

Theorem 2.11: Consistency of the system (30) is necessary for the existence of a nontrivial n-integral to the chain (1).

One can specify the system. Since

$$
\begin{gathered}
A_{N}^{(N+1)}=X_{1}\left\{C(N+1, N-1) g_{t_{-1}}-C(N+1, N) \frac{g_{t}}{g_{t_{x}}}\right\}=\frac{(N+1) N}{2} g_{t_{-1} t_{-1}}-(N+1) \frac{g_{t t_{-1}} g_{t_{x}}-g_{t} g_{t_{x} t_{-1}}}{g_{t_{x}}^{2}}, \\
A_{N+1}^{(N+1)}=\left\{C(N+1, N) g_{t_{-1}}-C(N+1, N+1) \frac{g_{t}}{g_{t_{x}}}\right\}=(N+1) g_{t_{-1}}-\frac{g_{t}}{g_{t_{x}}}, \\
A_{N}^{(N)}=\left\{C(N, N-1) g_{t_{-1}}-C(N, N) \frac{g_{t}}{g_{t_{x}}}\right\}=N g_{t_{-1}}-\frac{g_{t}}{g_{t_{x}}},
\end{gathered}
$$

the last equation of (30) becomes 


$$
\begin{aligned}
& \left\{\frac{(N+1) N}{2} g_{t_{-1} t_{-1}}-(N+1) \frac{g_{t t_{-1}} g_{t_{x}}-g_{t} g_{t_{x} t_{-1}}}{g_{t_{x}}^{2}}\right\}+\mu_{N}\left\{(N+1) g_{t_{-1}}-\frac{g_{t}}{g_{t_{x}}}\right\}=\mu_{N}\left\{N g_{t_{-1}}-\frac{g_{t}}{g_{t_{x}}}\right\} \\
& -D_{x}\left(\mu_{N}\right),
\end{aligned}
$$

that can be rewritten as

$$
\frac{(N+1) N}{2} g_{t_{-1} t_{-1}}-(N+1) \frac{g_{t t_{-1}} g_{t_{x}}-g_{t} g_{t_{x} t_{-1}}}{g_{t_{x}}^{2}}+\mu_{N} g_{t_{-1}}=-D_{x}\left(\mu_{N}\right) .
$$

If $C_{1}=0$ then, by Lemma 2.10,

$$
0=X_{1} D^{-1} Y_{0}(f)=\frac{\partial}{\partial t_{-1}} D^{-1}\left(f_{t_{1}}\right)
$$

\section{PROOF OF THEOREM 1.3}

\section{A. Case 1: $t_{1 x}=t_{x}+A\left(t_{1}-t\right)$}

Introduce $\tau=t_{1}-t$ and rewrite the equation as $\tau_{x}=A(\tau)$. Study the question when this equation admits a nontrivial $n$-integral or the same when the corresponding Lie algebra $L_{n}$ is of finite dimension. Since

$$
\begin{gathered}
Y_{0} f=A^{\prime}(\tau) \tau_{t_{1}}=D_{\tau} A(\tau), \\
Y_{0} f_{x}=A^{\prime \prime}(\tau) A(\tau)+A^{\prime}(\tau) A^{\prime}(\tau)=D_{\tau} A(\tau) D_{\tau} A(\tau),
\end{gathered}
$$

and $Y_{0} D_{x}^{k} f=\left(D_{\tau} A(\tau)\right)^{k+1}$, we can write $Y_{1}$ as

$$
Y_{1}=\frac{\partial}{\partial t}+\sum_{k=1}^{\infty} D^{-1}\left(D_{\tau} A(\tau)\right)^{k} \frac{\partial}{\partial D_{x}^{k} t} .
$$

Now let us introduce new variables: $\tau_{+}=t, \tau=t_{1}-t, \tau_{-1}=t-t_{-1}, \tau_{j}=t_{j+1}-t_{j}$. Since

$$
\frac{\partial}{\partial t}=\frac{\partial}{\partial \tau_{+}}-\frac{\partial}{\partial \tau}+\frac{\partial}{\partial \tau_{-1}}
$$

then the expression (33) for $Y_{1}$ becomes

$$
Y_{1}=\frac{\partial}{\partial \tau_{+}}-\frac{\partial}{\partial \tau}+\frac{\partial}{\partial \tau_{-1}}+\sum_{k=1}^{\infty} D^{-1}\left(D_{\tau} A(\tau)\right)^{k} \frac{\partial}{\partial D_{x}^{k} \tau_{+}} .
$$

One can ignore the term containing $\partial / \partial \tau$ since coefficients in the vector fields used below do not depend on $\tau$.

Multiply $Y_{1}$ by $A\left(\tau_{-1}\right)$,

$$
A\left(\tau_{-1}\right) Y_{1}=A\left(\tau_{-1}\right) \frac{\partial}{\partial \tau_{+}}+A\left(\tau_{-1}\right) \frac{\partial}{\partial \tau_{-1}}+\sum_{k=1}^{\infty} A\left(\tau_{-1}\right) D^{-1}\left(D_{\tau} A(\tau)\right)^{k} \frac{\partial}{\partial D_{x}^{k} \tau_{+}}
$$

Introduce

$$
p(\theta)=A\left(\tau_{-1}(\theta)\right), \quad \text { where } d \theta=\frac{d \tau_{-1}}{A\left(\tau_{-1}\right)} .
$$

Equation (35) becomes 


$$
A\left(\tau_{-1}\right) Y_{1}=p(\theta) \frac{\partial}{\partial \tau_{+}}+\frac{\partial}{\partial \theta}+\sum_{k=1}^{\infty} D_{x}^{k}(p(\theta)) \frac{\partial}{\partial D_{x}^{k} \tau_{+}} .
$$

Now instead of $X_{1}=\partial / \partial t_{-1}$, define

$$
\tilde{X}_{1}=A\left(\tau_{-1}\right) X_{1}=-A\left(\tau_{-1}\right) \frac{\partial}{\partial \tau_{-1}}+A\left(\tau_{-1}\right) \frac{\partial}{\partial \tau_{-2}} .
$$

It is indeed with new variables

$$
\tilde{X}_{1}=-\frac{\partial}{\partial \theta}+\frac{p(\theta)}{p\left(\theta_{-1}\right)} \frac{\partial}{\partial \theta_{-1}} .
$$

Note that $\left[D_{x}, \widetilde{X}_{1}\right]=D_{x}\left(p(\theta) / p\left(\theta_{-1}\right)\right) W_{1}$, where $W_{1}=\partial / \partial \theta_{-1}$. Since $\left[D_{x}, X_{1}\right]=-X_{1}(g) X_{1}-X_{1}\left(g_{-1}\right) X_{2}$, then $\left[D_{x}, \widetilde{X}_{1}\right] \in L_{n}$. Therefore, we have two possibilities:

(ii) $W_{1} \in L_{n}$.

First let us consider case (i). We have

$$
D_{x}\left(\frac{p(\theta)}{p\left(\theta_{-1}\right)}\right)=\frac{p^{\prime}(\theta) p\left(\theta_{-1}\right)-p(\theta) p^{\prime}\left(\theta_{-1}\right)}{p^{2}\left(\theta_{-1}\right)}=0 .
$$

Solving this differential equation we get $p(\theta)=A\left(\tau_{-1}(\theta)\right)=\mu e^{\lambda \theta}$. Since $d \theta / d \tau_{-1}=1 / A\left(\tau_{-1}\right)$, we have $A(\tau)=\lambda \tau+c$.

Now concentrate on case (ii). Since $D_{x}\left(p(\theta) / p\left(\theta_{-1}\right)\right) W_{1} \in L_{n}$, then $W_{1} \in L_{n}$ and, due to (38), $W=\partial / \partial \theta \in L_{n}$.

Lemma 3.1: If equation $\tau_{x}=A(\tau)$ admits a nontrivial $n$-integral then function $p(\theta)$, defined by (36), is a quasipolynomial.

Proof: Instead of $Y_{1}, X_{1}$, take the pair of the operators $W=\partial / \partial \theta$ and

$$
Z=A\left(\tau_{-1}\right) Y_{1}-W=p(\theta) \frac{\partial}{\partial \tau_{+}}+D_{x} p(\theta) \frac{\partial}{\partial \tau_{+x}}+D_{x}^{2}(p(\theta)) \frac{\partial}{\partial \tau_{+x x}}+\cdots
$$

Construct a sequence of the operators

$$
C_{1}=[W, Z], \quad C_{2}=\left[W, C_{1}\right], \quad C_{k}=\left[W, C_{k-1}\right], \quad k \geq 2 .
$$

Since algebra $L_{n}$ is of finite dimension then there exists number $N$, such that

$$
C_{N+1}=\mu_{0} Z+\mu_{1} C_{1}+\cdots+\mu_{N} C_{N},
$$

and vector fields $Z, C_{1}, \ldots, C_{N}$ are linearly independent.

Direct calculations show that $\left[D_{x}, W\right]=\left[D_{x}, Z\right]=0$. Therefore, we have $\left[D_{x}, C_{j}\right]=0$ for all $j$. It follows from (41) that

$$
0=D_{x}\left(\mu_{0}\right) Z+D_{x}\left(\mu_{1}\right) C_{1}+\cdots+D_{x}\left(\mu_{N}\right) C_{N},
$$

which implies $D_{x}\left(\mu_{j}\right)=0$. Clearly $\mu_{j}=\mu_{j}(\theta)$ and $D_{x}\left(\mu_{j}\right)=\mu_{j}^{\prime}(\theta)=0$. Hence $\mu_{j}$ is constant for all $j \geq 0$.

Look at the coefficients of $\partial / \partial \tau_{+}$in (41) and get

$$
\mu_{0} p(\theta)+\mu_{1} p^{\prime}(\theta)+\cdots+\mu_{N} p^{(N)}(\theta)=p^{(N+1)}(\theta) .
$$

This means $p(\theta)$ is a quasipolynomial, i.e., it takes the form 


$$
p(\theta)=\sum_{j=1}^{s} q_{j}(\theta) e^{\lambda_{j} \theta} .
$$

Lemma 3.2: Let $p(\theta)$ is an arbitrary quasipolynomial solving a differential equation of the form (42) and which does not solve any equation of this form of less order. Then the equation $t_{1 x}=t_{x}+A\left(t_{1}-t\right)$ with $A$ found from the conditions,

$$
\begin{gathered}
A\left(\tau_{-1}\right)=p(\theta), \\
\tau_{-1}=\int_{0}^{\theta} p(\tilde{\theta}) d \tilde{\theta}
\end{gathered}
$$

admits a nontrivial n-integral.

Proof: Introduce

$$
L\left(D_{x}\right)=D_{x}^{N+1}-\mu_{N} D_{x}^{N}-\mu_{N-1} D_{x}^{N-1}-\cdots-\mu_{1} D_{x}-\mu_{0} .
$$

Equation (42) can be rewritten as $L\left(D_{x}\right) p(\theta)=0$. However, $L\left(D_{x}\right) p(\theta)=L\left(D_{x}\right) A\left(\tau_{-1}\right)$. Since $L\left(D_{x}\right) t_{1 x}=L\left(D_{x}\right) t_{x}+L\left(D_{x}\right) A(\tau)$ and $L\left(D_{x}\right) A(\tau)=0$, we have $L\left(D_{x}\right) t_{1 x}=L\left(D_{x}\right) t_{x}$. But $L\left(D_{x}\right) t_{1 x}$ $=D L\left(D_{x}\right) t_{x}$, therefore $D L\left(D_{x}\right) t_{x}=L\left(D_{x}\right) t_{x}$. Denote $L\left(D_{x}\right) t_{x}=I$ so we have $D I=I$. Hence $L\left(D_{x}\right) t_{x}$ is an $n$-integral.

Therefore the condition (43) is necessary and sufficient for our equation to have nontrivial $n$-integral.

Example 1: Take $p(\theta)=\frac{1}{2} e^{\theta}+\frac{1}{2} e^{-\theta}=\cosh \theta$, then

$$
\begin{aligned}
& A\left(\tau_{-1}\right)=\cosh \theta, \\
& \tau_{-1}=\sinh \theta+c,
\end{aligned}
$$

or $A\left(\tau_{-1}\right)^{2}-\left(\tau_{-1}-c\right)^{2}=1$ which gives $A\left(\tau_{-1}\right)=\sqrt{1+\left(\tau_{-1}-c\right)^{2}}$. So $t_{1 x}=t_{x}+\sqrt{1+\left(t_{1}-t-c\right)^{2}}$, where $c$ is arbitrary constant, is Darboux integrable. Moreover, its general solution is given by $t(n, x)$ $=G(x)+n c+\sum_{k=0}^{n-1} \sinh \left(x+c_{k}\right)$, where $G(x)$ is arbitrary function depending on $x$ and $c_{k}$ are arbitrary constants.

\section{B. Case 2: $t_{1 x}=t_{x}+c_{1}\left(t_{1}-t\right) t+c_{2}\left(t_{1}-t\right)^{2}+c_{3}\left(t_{1}-t\right)$}

Lemma 3.3: If equation $t_{1 x}=t_{x}+d\left(t, t_{1}\right)=t_{x}+c_{1}\left(t_{1}-t\right) t+c_{2}\left(t_{1}-t\right)^{2}+c_{3}\left(t_{1}-t\right)$ admits a nontrivial $n$-integral, then there exists a natural number $k$ such that

$$
k c_{1}-(k+1) c_{2}=0 .
$$

Proof: Introduce vector fields $T_{1}=\left[X_{1}, Y_{1}\right], T_{m}=\left[X_{1}, T_{m-1}\right], m \geq 2$. Direct calculations show that

$$
\begin{gathered}
{\left[D_{x}, T_{1}\right]=\left(-c_{1}+2 c_{2}\right) X_{1}+\left(-c_{1}+2 c_{2}\right) Y_{1}+\left(d_{t_{-1}}\left(t_{-1}, t\right)-d_{t}\left(t_{-1}, t\right)\right) T_{1},} \\
{\left[D_{x}, T_{m}\right]=-A_{m-1}^{(m)} T_{m-1}-A_{m}^{(m)} T_{m},}
\end{gathered}
$$

where

$$
A_{j}^{(m)}=X_{1}^{m-j}\left\{-C(m, j-1) d_{t_{-1}}\left(t_{-1}, t\right)+C(m, j) d_{t}\left(t_{-1}, t\right)\right\}, \quad C(m, k)=\frac{m !}{k !(m-k) !} .
$$

Due to finiteness of algebra $L_{n}$, there exists natural number $M$, such that 


$$
T_{M+1}=\mu_{1} T_{1}+\mu_{2} T_{2}+\cdots+\mu_{M} T_{M},
$$

and $T_{1}, T_{2}, \ldots, T_{M}$ are linearly independent. We have

$$
\left[D_{x}, T_{M+1}\right]=\left[D_{x}, \mu_{1} T_{1}+\mu_{2} T_{2}+\cdots+\mu_{M} T_{M}\right],
$$

that can be rewritten by (45) in the following form:

$$
\begin{aligned}
& -A_{M}^{(M+1)} T_{M}-A_{M+1}^{(M+1)}\left\{\mu_{M} T_{M}+\mu_{M-1} T_{M-1}+\ldots+\mu_{1} T_{1}\right\} \\
& \quad=D_{x}\left(\mu_{1}\right) T_{1}-\mu_{1}\left(c_{1}-2 c_{2}\right) X_{1}-\mu_{1}\left(c_{1}-2 c_{2}\right) Y_{1}-\mu_{1} A_{1}^{(1)} T_{1}+D_{x}\left(\mu_{2}\right) T_{2}-\mu_{2} A_{1}^{(2)} T_{1}-\mu_{2} A_{2}^{(2)} T_{2} \\
& \quad+\cdots+D_{x}\left(\mu_{M}\right) T_{M}-\mu_{M} A_{M-1}^{(M)} T_{M-1}-\mu_{M} A_{M}^{(M)} T_{M} .
\end{aligned}
$$

Compare coefficients before the operators. The coefficient before $X_{1}$ and $Y_{1}$ gives $-\mu_{1}\left(c_{1}-2 c_{2}\right)$ $=0$. In this case we have two choices: $\mu_{1}=0$ or $c_{1}-2 c_{2}=0$. The second one gives (44) with $k$ $=1$. If $c_{1}-2 c_{2} \neq 0$, then $\mu_{1}=0$. Using this, from the coefficient of $T_{1}$ we get $-\mu_{2} A_{1}^{(2)}=0$. Again, we have that either $\mu_{2}=0$ or $A_{1}^{(2)}=0$. If $A_{1}^{(2)}=0$ we stop, if not then $\mu_{2}=0$ and we continue to compare the coefficients. Using $\mu_{1}=\mu_{2}=0$, the coefficient before $T_{2}$ gives $-\mu_{3} A_{2}^{(3)}=0$ which means $\mu_{3}$ $=0$ or $A_{2}^{(3)}=0$. Same as before: if $A_{2}^{(3)}=0$, we stop, if not then $\mu_{3}=0$ and we continue to the procedure.

If $\mu_{1}=\cdots=\mu_{M}=0$ then $T_{M+1}=0$ and $\left[D_{x}, T_{M+1}\right]=0=-A_{M}^{(M+1)} T_{M}-A_{M+1}^{(M+1)} T_{M+1}=-A_{M}^{M+1} T_{M}$. Since $T_{1}, \ldots, T_{M}$ are linearly independent then $T_{M} \neq 0$ and therefore $A_{M}^{(M+1)}=0$. It follows $A_{k-1}^{(k)}=0$ for some $k=1,2, \ldots, M+1$. Evaluate $A_{k-1}^{(k)}$,

$$
\begin{aligned}
A_{k-1}^{(k)}= & -C(k, k-2) d_{t_{-1} t_{-1}}\left(t_{-1}, t\right)+C(k, k-1) d_{t t_{-1}}\left(t_{-1}, t\right)=-k(k-1)\left(c_{2}-c_{1}\right)+k\left(c_{1}-2 c_{2}\right)=k\left\{k c_{1}\right. \\
& \left.-(k+1) c_{2}\right\} .
\end{aligned}
$$

Let us rewrite the equation in case (2) as

$$
\tau_{x}=c_{1} \tau t+c_{2} \tau^{2}+c_{3} \tau
$$

where $\tau=t_{1}-t$. We have two important relations.

(1) $Y_{0} f=D_{x} \ln H$, where

$$
H=\frac{\tau \theta^{1 / \epsilon}}{(\theta+\epsilon)^{1 / \epsilon}}, \quad \theta=\frac{\tau_{1}}{\tau}, \quad \epsilon=\frac{c_{1}}{c_{2}}-1 .
$$

(2) $Y_{1} f=D_{x} \ln R H_{-1}$, where

$$
H_{-1}=D^{-1} H, \quad R=\frac{\theta}{\tau(\theta+\epsilon)}, \quad \text { when } \epsilon \neq 0 .
$$

[The case $\epsilon=0$, i.e $c_{1}=c_{2}$, is not realized due to Lemma 3.3. The case $c_{2}=0$, due to Lemma 3.3 , leads to $c_{1}=0$, and the equation becomes $t_{1 x}=t_{x}+c_{3}\left(t_{1}-t\right)$ with an $n$-integral $I=t_{x}-c_{3} t$.]

These two relations allow us to simplify the basis operators $Y_{0}, Y_{1}, X_{1}$. Really, we take

$$
\tilde{Y}_{1}=H_{-1} Y_{1}, \quad \tilde{Y}_{0}=H Y_{0},
$$

and get $\left[D_{x}, \tilde{Y}_{0}\right]=0$ and $\left[D_{x}, \tilde{Y}_{1}\right]=\Lambda \tilde{Y}_{0}$, where $\Lambda=-\left(H_{-1} / H\right) D_{x} \ln \left(R H_{-1}\right)$.

First we will restrict the set of the variables as follows: $t_{1}, t, t_{-1}, t_{x}, t_{x x}, \ldots$ and change the variables $t^{+}=t, \tau_{-1}=t-t_{-1}$ keeping the other variables unchanged. Then some of the differentiations will change, 


$$
\frac{\partial}{\partial t}=\frac{\partial}{\partial t^{+}}+\frac{\partial}{\partial \tau_{-1}}, \quad \frac{\partial}{\partial t_{-1}}=-\frac{\partial}{\partial \tau_{-1}} .
$$

So we have $X_{1}=-\partial / \partial \tau_{-1}=-\hat{X}_{1}$ and

$$
\tilde{Y}_{1}=H_{-1}\left(\frac{\partial}{\partial t^{+}}+\frac{\partial}{\partial \tau_{-1}}\right)+\sum_{k=1}^{\infty} H_{-1} D^{-1}\left(Y_{0} D_{x}^{k-1} f\right) \frac{\partial}{\partial t_{[k]}}
$$

Since $\left[D_{x}, \hat{X}_{1}\right]=D_{x}\left(\ln R_{-1}\right) \hat{X}_{1}$, one can introduce $\tilde{X}_{1}=\left(1 / R_{-1}\right) \hat{X}_{1}$ and get $\left[D_{x}, \tilde{X}_{1}\right]=0$. Here $R_{-1}$ $=D^{-1} R$.

Introduce vector fields $C_{2}=\left[\tilde{X}_{1}, \tilde{Y}_{1}\right], C_{3}=\left[\tilde{X}_{1}, C_{2}\right], C_{k}=\left[\tilde{X}_{1}, C_{k-1}\right], k \geq 3$. We have

$$
\left[D_{x}, C_{j+1}\right]=\tilde{X}_{1}^{j}(\Lambda) \tilde{Y}_{0}, \quad j \geq 1 .
$$

Since the algebra $L_{n}$ is of finite dimension then there is a number $N$, such that

$$
C_{N+1}=\mu_{N} C_{N}+\cdots+\mu_{2} C_{2}+\mu_{1} \tilde{Y}_{1},
$$

where $\tilde{Y}_{1}, C_{1}, C_{2}, \ldots$ are linearly independent.

Applying the commutator with $D_{x}$ one gets $D_{x}\left(\mu_{j}\right)=0$ for $j=1, \ldots, N$ and

$$
\left(\tilde{X}_{1}^{N}-\mu_{N} \tilde{X}_{1}^{N-1}-\cdots-\mu_{1}\right) \Lambda=0 .
$$

All the operators in our sequence have coefficients depending on $\tau, \tau_{-1}$, $t$. So do $\mu_{j}$ $=\mu_{j}\left(\tau, \tau_{-1}, t\right)$. But the relation $D_{x} \mu_{j}\left(\tau, \tau_{-1}, t\right)=0$ shows that $\partial \mu_{j} / \partial t=0$, i.e., $\mu_{j}=\mu_{j}\left(\tau, \tau_{-1}\right)$. Since the minimal $x$-integral for an equation in case (2) depends on variables $t, t_{1}, t_{2}, t_{3}$, the relation $D_{x}\left(\mu_{j}\right)=0$ implies that $\mu_{j}$ is constant for all $j$.

Introduce new variables $\widetilde{t}_{1}, \widetilde{t}, \eta$ as

$$
\begin{gathered}
\tilde{t}_{1}=t_{1}, \quad \tilde{t}=t^{+} \\
\eta=\ln \left(\frac{\tau_{-1}}{\tau_{-1}+\frac{1}{\epsilon}\left(t_{1}-t^{+}\right)}\right), \quad \text { or the same } \tau_{-1}=\frac{\tau}{\epsilon}\left(\frac{e^{\eta}}{1-e^{\eta}}\right) .
\end{gathered}
$$

Then

$$
\begin{gathered}
\frac{\partial}{\partial \tau_{-1}}=\frac{\partial \eta}{\partial \tau_{-1}} \frac{\partial}{\partial \eta}, \\
\frac{\partial}{\partial t^{+}}=\frac{\partial}{\partial \widetilde{t}^{+}}+\frac{\partial \eta}{\partial t^{+}} \frac{\partial}{\partial \eta}, \\
\frac{\partial}{\partial t_{1}}=\frac{\partial}{\partial \widetilde{t}_{1}}+\frac{\partial \eta}{\partial t_{1}} \frac{\partial}{\partial \eta} .
\end{gathered}
$$

In these new variables $\tilde{X}_{1}$ takes the form

$$
\tilde{X}_{1}=\frac{\tau_{-1}\left(\theta_{-1}+\epsilon\right)}{\theta_{-1}} \frac{\partial}{\partial \tau_{-1}}=\frac{\partial}{\partial \eta}
$$

and Eq. (48) becomes 


$$
\left(\frac{d^{N}}{d \eta^{N}}-\mu_{N} \frac{d^{N-1}}{d \eta^{N-1}}-\cdots-\mu_{1}\right) \Lambda=0
$$

where

$$
\Lambda=-\frac{H_{-1}}{H}\left(\tau_{x} \ln R+D_{x} \ln H_{-1}\right)=-\frac{H_{-1}}{H}\left(\frac{\partial f}{\partial t}+D^{-1} \frac{\partial f}{\partial t_{1}}\right)=-\frac{H_{-1}}{H}\left(c_{1}-2 c_{2}\right)\left(\tau-\tau_{-1}\right) .
$$

Let us show that $c_{1}-2 c_{2}=0$. Assume contrary. It follows from (50) and (51) that both functions $H_{-1}$ and $\tau_{-1} H_{-1}$ should solve the linear differential equation with constant coefficients,

$$
\left(\frac{d^{N}}{d \eta^{N}}-\mu_{N} \frac{d^{N-1}}{d \eta^{N-1}}-\cdots-\mu_{1}\right) y(\eta)=0
$$

Therefore, both functions $H_{-1}$ and $\tau_{-1} H_{-1}$ must be quasipolynomials in $\eta$.

Due to (46) and (49), we have

$$
H_{-1}=\frac{\tau}{\epsilon} e^{\eta}\left(1-e^{\eta}\right)^{1 / \epsilon-1}
$$

and

$$
\tau_{-1} H_{-1}=\frac{\tau^{2}}{\epsilon^{2}} e^{2 \eta}\left(1-e^{\eta}\right)^{1 / \epsilon-2}
$$

To be quasipolynomials in $\eta$ it is necessary that $\epsilon=1 / m$ for some natural $m \geq 2$.

Rewrite our vector fields $\tilde{X}_{1}, \tilde{Y}_{1}$ in the new variables;

$$
\begin{gathered}
\tilde{X}_{1}=\frac{\partial}{\partial \eta} \\
\tilde{Y}_{1}=H_{-1} \frac{\partial}{\partial \widetilde{t}}+H_{-1}\left(\frac{\partial \eta}{\partial t^{+}}+\frac{\partial \eta}{\partial \tau_{-1}}\right) \frac{\partial}{\partial \eta}+\cdots
\end{gathered}
$$

Study the projection on the direction $\partial / \partial \eta$.

The operators $\tilde{X}_{1}=\partial / \partial \eta$ and $H_{-1}\left(\partial \eta / \partial t^{+}+\partial \eta / \partial \tau_{-1}\right) \partial / \partial \eta$ generate a finite dimensional Lie algebra over the field of constants. Due to Lemma 2.3 in this case the coefficient $H_{-1} \partial \eta / \partial t$ should be of one of the forms

$$
\tilde{c}_{1} e^{\widetilde{\alpha} \eta}+\widetilde{c}_{2} e^{-\widetilde{\alpha} \eta}+\tilde{c}_{3} \quad \text { or } \quad \tilde{c}_{1} \eta^{2}+\widetilde{c}_{2} \eta+\widetilde{c}_{3} \text {, }
$$

but we have

$$
H_{-1}\left(\frac{\partial \eta}{\partial t^{+}}+\frac{\partial \eta}{\partial \tau_{-1}}\right)=\left(1+\left(\frac{1}{\epsilon}-1\right) e^{\eta}\right)\left(1-e^{\eta}\right)^{1 / \epsilon}
$$

with $1 / \epsilon=m \geq 2$ and it is never of the form (52). This contradiction shows that $c_{1}-2 c_{2}=0$.

\section{Case 3: $t_{1 x}=t_{x}+A\left(t_{1}-t\right) e^{\alpha t}$}

Introduce $\tau=t_{1}-t$ and rewrite the equation as $\tau_{x}=A(\tau) e^{\alpha t}$. Study the question when the equation admits a nontrivial $n$-integral or the same when the corresponding Lie algebra $L_{n}$ is of finite dimension.

Instead of the vector fields $Y_{0}=\partial / \partial t_{1}$ and $Y_{1}=\partial / \partial t+D^{-1}\left(\partial f / \partial t_{1}\right)\left(\partial / \partial t_{x}\right)+D^{-1}\left(\partial f_{x} / \partial t_{1}\right)\left(\partial / \partial t_{x x}\right)$ $+\cdots$, we will use the vector fields $\tilde{Y}_{0}=A(\tau) Y_{0}$ and $\tilde{Y}_{1}=A\left(\tau_{-1}\right) Y_{1}$. They are more convenient since they satisfy more simple relations, 


$$
\left[D_{x}, \tilde{Y}_{0}\right]=0, \quad\left[D_{x}, \tilde{Y}_{1}\right]=\lambda_{1} \tilde{Y}_{0}
$$

as operators acting on the enlarged set $t_{1}, t, t_{-1}, t_{-2}, \ldots ; t_{x}, t_{x x}, t_{x x x}, \ldots$ Here the coefficient $\lambda_{1}$ is

$$
\lambda_{1}=\frac{A\left(\tau_{-1}\right)}{A(\tau)}\left(A^{\prime}(\tau)-\alpha A(\tau)-A^{\prime}\left(\tau_{-1}\right) e^{\left.-\alpha \tau_{-1}\right)} e^{\alpha t} .\right.
$$

Since the equation is represented as $\tau_{x}=A(\tau) e^{\alpha t}$ it is reasonable to introduce new variables as $\tau_{+}$ $=t, \tau_{-1}=t-t_{-1}, \tau_{-2}=t_{-1}-t_{-2}$, such that

$$
\frac{\partial}{\partial t}=\frac{\partial}{\partial \tau_{+}}+\frac{\partial}{\partial \tau_{-1}}, \quad \frac{\partial}{\partial t_{-1}}=-\frac{\partial}{\partial \tau_{-1}}+\frac{\partial}{\partial \tau_{-2}}, \quad \frac{\partial}{\partial t_{-2}}=-\frac{\partial}{\partial \tau_{-2}} .
$$

Instead of the operators $X_{1}=\partial / \partial t_{-1}$ and $X_{2}=\partial / \partial t_{-2}$ use new ones $\tilde{X}_{1}=A\left(\tau_{-1}\right) e^{-\alpha \tau_{-1}} \partial / \partial \tau_{-1}$ and $\tilde{X}_{2}$ $=A\left(\tau_{-2}\right) e^{-\alpha \tau_{-2}} \partial / \partial \tau_{-2}$. They satisfy relations $\left[D_{x}, \tilde{X}_{2}\right]=0$ and $\left[D_{x}, \widetilde{X}_{1}\right]=\mu \tilde{X}_{2}$. Here the coefficient $\mu$ is

$$
\mu=\alpha A\left(\tau_{-1}\right) e^{-2 \alpha \tau_{-1}+\alpha t} .
$$

Construct a sequence by taking $\tilde{X}_{1}, \tilde{Y}_{1}, C_{2}=\left[\tilde{X}_{1}, \tilde{Y}_{1}\right], C_{3}=\left[\tilde{X}_{1}, C_{2}\right], C_{k}=\left[\tilde{X}_{1}, C_{k-1}\right]$ for $k \geq 3$. One can easily check that

$$
\begin{gathered}
{\left[D_{x}, C_{2}\right]=-\tilde{Y}_{1}(\mu) \tilde{X}_{2}+\tilde{X}_{1}\left(\lambda_{1}\right) \tilde{Y}_{0}=b_{2} \tilde{X}_{2}+\tilde{X}_{1}\left(\lambda_{1}\right) \tilde{Y}_{0},} \\
{\left[D_{x}, C_{3}\right]=\tilde{X}_{1}^{2}\left(\lambda_{1}\right) \tilde{Y}_{0}-\left(C_{2}+\tilde{X}_{1} \tilde{Y}_{1}\right)(\mu) \tilde{X}_{2}=\tilde{X}_{1}^{2}\left(\lambda_{1}\right) \tilde{Y}_{0}+b_{3} \tilde{X}_{2},}
\end{gathered}
$$

and for any $k$ (it can be proven by induction)

$$
\left[D_{x}, C_{k}\right]=\widetilde{X}_{1}^{k-1}\left(\lambda_{1}\right) \tilde{Y}_{0}+b_{k} \tilde{X}_{2} .
$$

Since the characteristic Lie algebra $L_{n}$ is of finite dimension then there is a number $N$, such that

$$
C_{N+1}=\mu_{N} C_{N}+\cdots+\mu_{1} \tilde{Y}_{1}+\mu_{0} \tilde{X}_{1},
$$

where $\tilde{X}_{1}, \tilde{Y}_{1}, C_{1}, C_{2}, \ldots$ are linearly independent.

Commute both sides of (53) with $D_{x}$ and get

$$
\begin{aligned}
\tilde{X}_{1}^{N}\left(\lambda_{1}\right) \tilde{Y}_{0}+b_{N+1} \tilde{X}_{2}= & D_{x}\left(\mu_{N}\right) C_{N}+\cdots+D_{x}\left(\mu_{1}\right) \tilde{Y}_{1}+D_{x}\left(\mu_{0}\right) \tilde{X}_{1}+\mu_{N} \tilde{X}_{1}^{N-1}\left(\lambda_{1}\right) \tilde{Y}_{0}+\cdots+\mu_{1} \lambda_{1} \tilde{Y}_{0} \\
& +\left\{\sum_{k=2}^{N} b_{k} \mu_{k}\right\} \tilde{X}_{2} .
\end{aligned}
$$

Collect the coefficients before the operators and get $D_{x}\left(\mu_{j}\right)=0$ for $j=0,1, \ldots, N$, and

$$
\left(\widetilde{X}_{1}^{N}-\mu_{N} \widetilde{X}_{1}^{N-1}-\mu_{N-1} \tilde{X}_{1}^{N-2}-\cdots-\mu_{1}\right) \lambda_{1}=0 .
$$

Introduce new variables $\eta, \eta_{-1}$ as solutions of the following ordinary differential equations:

$$
\frac{d \tau_{-1}}{d \eta}=A\left(\tau_{-1}\right) e^{-\alpha \tau_{-1}}, \quad \frac{d \tau_{-2}}{d \eta_{-1}}=A\left(\tau_{-2}\right) e^{-\alpha \tau_{-2}} .
$$

Thus our vector fields are rewritten as

$$
\tilde{X}_{1}=\frac{\partial}{\partial \eta}, \quad \tilde{X}_{2}=\frac{\partial}{\partial \eta_{-1}}, \quad \tilde{Y}_{0}=A(\tau) \frac{\partial}{\partial t_{1}},
$$




$$
\tilde{Y}_{1}=e^{\alpha \tau_{-1}} \frac{\partial}{\partial \eta}+A\left(\tau_{-1}\right) \frac{\partial}{\partial \tau_{+}}+D_{x}\left(A\left(\tau_{-1}\right)\right) \frac{\partial}{\partial t_{x}}+\cdots .
$$

By looking at the projection on $\partial / \partial \eta$ we get an algebra generated by $\partial / \partial \eta$ and $e^{\alpha \tau_{-1}} \partial / \partial \eta$ containing all possible commutators and all possible linear combinations with constant coefficients. Due to Lemma 2.3, we get that $e^{\alpha \tau_{-1}}$ can be only one of the forms (a) $e^{\alpha \tau_{-1}}=c_{1} e^{\beta \eta}+c_{2} e^{-\beta \eta}+c_{3}$ and (b) $e^{\alpha \tau_{-1}}=c_{1} \eta^{2}+c_{2} \eta+c_{3}$,

where $\beta, c_{1}, c_{2}, c_{3}$ are some constants.

The equation $A\left(\tau_{-1}\right)=(1 / \alpha)(d / d \eta) e^{\alpha \tau_{-1}}$ implies that in case (a) we have $A\left(\tau_{-1}\right)=(\beta / \alpha)$ $\times\left(c_{1} e^{\beta \eta}-c_{2} e^{-\beta \eta}\right)$, or the same

$$
A^{2}(\tau)=\frac{\beta^{2}}{\alpha^{2}}\left\{\left(e^{\alpha \tau}-c_{3}\right)^{2}-4 c_{1} c_{2}\right\},
$$

and in case (b) we have $A\left(\tau_{-1}\right)=(1 / \alpha)\left(2 c_{1} \eta+c_{2}\right)$, or the same,

$$
A^{2}(\tau)=\frac{4 c_{1}}{\alpha^{2}} e^{\alpha \tau}+\frac{c_{2}^{2}-4 c_{1} c_{3}}{\alpha^{2}}
$$

In addition to the operators $\tilde{X}_{1}, \widetilde{X}_{2}, \widetilde{Y}_{0}, \widetilde{Y}_{1}$ introduced above, we will use $\widetilde{Y}_{2}$ $=A\left(\tau_{-2}\right) D^{-1}\left(Y_{1} f\right) \partial_{t_{x}}+A\left(\tau_{-2}\right) D^{-1}\left(Y_{1} f_{x}\right) \partial_{t_{x x}}+\cdots$ defined as $\tilde{Y}_{2}=A\left(\tau_{-2}\right) Y_{2}$. It satisfies the commutativity relation

$$
\left[D_{x}, \widetilde{Y}_{2}\right]=\lambda \widetilde{Y}_{1}+\xi \widetilde{Y}_{0}+\nu \widetilde{X}_{1}
$$

where

$$
\begin{gathered}
\xi=-\frac{A\left(\tau_{-2}\right)}{A(\tau)} D^{-1}\left(Y_{1} f\right)=-\frac{A\left(\tau_{-2}\right)}{A(\tau)}\left\{\left(-A^{\prime}\left(\tau_{-1}\right)+\alpha A\left(\tau_{-1}\right)\right) e^{-\alpha \tau_{-1}}+A^{\prime}\left(\tau_{-2}\right) e^{\left.-\alpha \tau_{-2}-\alpha \tau_{-1}\right\}} e^{\alpha t},\right. \\
\lambda=-\frac{A\left(\tau_{-2}\right)}{A\left(\tau_{-1}\right)} D^{-1}\left(Y_{1} f\right) \quad \text { and } \quad \nu=-\lambda e^{\alpha \tau_{-1}} .
\end{gathered}
$$

Lemma 3.4:

(1) Equation $t_{1 x}=t_{x}+(\beta / \alpha)\left(e^{\alpha \tau}-c_{3}\right) e^{\alpha t}$ admits a nontrivial $n$-integral if and only if $c_{3}= \pm 1$.

(2) Equation $t_{1 x}=t_{x}+c_{5} e^{\alpha t}, c_{5} \neq 0$ does not admit a nontrivial $n$-integral.

Proof: In this case the equation $\tau_{x}=A(\tau) e^{\alpha t}$ is reduced by evident scaling of $x$ and $t$ to

$$
t_{1 x}=t_{x}+e^{t} \quad \text { or } \quad t_{1 x}=t_{x}+e^{t_{1}}+\varepsilon e^{t} .
$$

By induction on $m$ one can easily see that for the equation $t_{1 x}=t_{x}+e^{t}$, the basic vector fields $Y_{m}$ are

$$
\begin{aligned}
& Y_{1}=\frac{\partial}{\partial t}, \\
& Y_{m}=e^{t_{-}(m-1)} \frac{\partial}{\partial t_{x}}+e^{t_{-}(m-1)}\left(t_{x}-e^{\left.t_{-}(m-1)\right)} \frac{\partial}{\partial t_{x x}}+\cdots .\right.
\end{aligned}
$$

Since these vector fields $Y_{m}, m \geq 1$, are linearly independent then equation $t_{1 x}=t_{x}+e^{t}$ does not admit a nontrivial $n$-integral.

For equation $t_{1 x}=t_{x}+e^{t_{1}}+\varepsilon e^{t}$, the basic vector fields $Y_{m}$ are 


$$
\begin{gathered}
Y_{1}=\frac{\partial}{\partial t}+e^{t} \frac{\partial}{\partial t_{x}}+e^{t}\left(t_{x}+e^{t}\right) \frac{\partial}{\partial t_{x x}}+\cdots \\
Y_{m}=(\varepsilon+1) e^{t_{-(m-1)}} \frac{\partial}{\partial t_{x}}+(\varepsilon+1) e^{t_{-(m-1)}}\left(t_{x}+(1-\varepsilon) e^{\left.t_{-(m-1)}\right)} \frac{\partial}{\partial t_{x x}}+\cdots\right.
\end{gathered}
$$

One can see that vector fields $Y_{m}, m \geq 1$, are linearly independent if $\varepsilon \neq \pm 1$. Therefore, if $\varepsilon$ $\neq \pm 1$, equation $t_{1 x}=t_{x}+e^{t_{1}}+\varepsilon e^{t}$ does not admit a nontrivial $n$-integral. If $\varepsilon=-1$, the equation becomes $t_{1 x}=t_{x}+e^{t_{1}}-e^{t}$, and one of its $n$-integrals is $I=t_{x}-e^{t}$. If $\varepsilon=1$, the equation becomes $t_{1 x}$ $=t_{x}+e^{t_{1}}+e^{t}$, and one of its $n$-integrals is $I=2 t_{x x}-t_{x}^{2}-e^{2 t}$.

Lemma 3.5: Let equation $t_{1 x}=t_{x}+A\left(t_{1}-t\right) e^{\alpha t}$ with $(a) A^{2}(\tau)=\left(\beta^{2} / \alpha^{2}\right)\left\{\left(e^{\alpha \tau}-c_{3}\right)^{2}-4 c_{1} c_{2}\right\}$ or $(b)$ $A^{2}(\tau)=\left(4 c_{1} / \alpha^{2}\right) e^{\alpha \tau}+\left(c_{2}^{2}-4 c_{1} c_{3}\right) / \alpha^{2}$, admit a nontrivial $n$-integral. Then in case $(a)$, we have, $A\left(t_{1}-t\right)=(\beta / \alpha) \sqrt{\left(e^{\alpha\left(t_{1}-t\right)}-c_{3}\right)^{2}-c_{3}^{2}+1}$, where $c_{3}$ is an arbitrary constant, and in case $(b)$, we have $A\left(t_{1}-t\right)=c e^{(\alpha / 2)\left(t_{1}-t\right)}$, where $c$ is an arbitrary constant.

In cases $(a)$ and $(b)$ the corresponding $n$-integrals are $I=(\alpha / 2) t_{x}^{2}-t_{x x}+(\alpha / 2) e^{2 \alpha t}$ and $I=$ $-(\alpha / 2) t_{x}^{2}+t_{x x}$

Proof: Note that

$$
D_{x} \rho=\lambda, \quad \text { where } \rho=-\frac{A\left(\tau_{-2}\right)}{A\left(\tau_{-1}\right)}-e^{\alpha \tau_{-2}} .
$$

This implies that the vector field,

$$
R_{2}=\tilde{Y}_{2}-\rho \tilde{Y}_{1},
$$

satisfies very simple and convenient relation,

$$
\left[D_{x}, R_{2}\right]=\widetilde{\xi} \widetilde{Y}_{0}+\nu \widetilde{X}_{1}, \quad \widetilde{\xi}=-\frac{A\left(\tau_{-2}\right)}{A(\tau)} D^{-1}\left(Y_{1} f\right)-\rho \lambda_{1}, \quad \nu=e^{\alpha \tau_{-1}} \frac{A\left(\tau_{-2}\right)}{A\left(\tau_{-1}\right)} D^{-1}\left(Y_{1} f\right)
$$

Study now the sequence

$$
R_{j+1}=\left[\hat{X}, R_{j}\right], \quad j \geq 2, \quad \text { where } \hat{X}=\tilde{X}_{1}+e^{-\alpha \tau_{-1}} X_{2} .
$$

Direct calculations show that

$$
\left[D_{x}, R_{m}\right]=\hat{X}^{(m-2)}(\widetilde{\xi}) \tilde{Y}_{0}+\hat{X}^{(m-2)}(\widetilde{\nu}) \tilde{X}_{1}+b_{m} \tilde{X}_{2} .
$$

Since $\tilde{X}_{1}, \widetilde{X}_{2}, \tilde{Y}_{0}, R_{2}$ are linearly independent, then there exists a number $N \geq 2$, such that

$$
R_{N+1}=\mu_{N} R_{N}+\mu_{N-1} R_{N-1}+\cdots \mu_{2} R_{2}+\mu_{1} \tilde{X}_{1}
$$

and

$$
\left[D_{x}, R_{N+1}\right]=\left[D_{x}, \mu_{N} R_{N}+\mu_{N-1} R_{N-1}+\cdots \mu_{2} R_{2}+\mu_{1} \tilde{X}_{1}\right] .
$$

We use $\left[D_{x}, \tilde{X}_{1}\right]=\alpha A\left(\tau_{-1}\right) e^{-2 \alpha \tau_{-1}+\alpha t} \tilde{X}_{2},\left[D_{x}, X_{2}\right]=0$, and (60) to compare the coefficients before linearly independent vector fields $R_{k}$ and $\tilde{Y}_{0}$ in (61). We have, $D_{x}\left(\mu_{k}\right)=0, k=2,3, \ldots, N$, and

$$
\hat{X}^{(N-1)}(\tilde{\xi})=\mu_{N} \hat{X}^{(N-2)}(\tilde{\xi})+\cdots+\mu_{2} \tilde{\xi}
$$

Under the change in variables 


$$
\eta=z, \quad \eta_{-1}=z_{-1}-q(z), \quad \frac{\partial q(z)}{\partial z}=-e^{-\alpha \tau_{-1}},
$$

equation (62) is reduced to

$$
\left(D_{z}^{N-1}-\mu_{N} D_{z}^{N-2}-\cdots-\mu_{2}\right) \tilde{\xi}=0,
$$

where $\quad \mu_{k}=\mu_{k}\left(\tau_{-1}, \tau_{-2}\right)=\mu_{k}\left(z, z_{-1}\right)$. Since $\quad D_{x}\left(z_{-1}\right)=0, \quad D_{x}(z)=e^{\alpha t} \neq 0$, and $\quad 0=D_{x}\left(\mu_{k}\right)$ $=D_{z_{-1}}\left(\mu_{k}\right) D_{x}\left(z_{-1}\right)+D_{z}\left(\mu_{k}\right) D_{x}(z)$, then coefficients $\mu_{k}$ do not depend on variable $z$. Since, due to (63),

$$
\begin{aligned}
\widetilde{\xi}= & -\frac{A\left(\tau_{-2}\right)}{A(\tau)} e^{-\alpha \tau_{-1}} e^{\alpha t}\left\{-A^{\prime}\left(\tau_{-1}\right)+\alpha A\left(\tau_{-1}\right)+A^{\prime}\left(\tau_{-2}\right) e^{\left.-\alpha \tau_{-2}\right\}}+\frac{A\left(\tau_{-2}\right)}{A(\tau)} e^{\alpha t}\left\{A^{\prime}(\tau)-\alpha A(\tau)\right.\right. \\
& \left.-A^{\prime}\left(\tau_{-1}\right) e^{-\alpha \tau_{-1}}\right\}+\frac{A\left(\tau_{-1}\right)}{A(\tau)} e^{\alpha \tau_{-2}} e^{\alpha t}\left\{A^{\prime}(\tau)-\alpha A(\tau)-A^{\prime}\left(\tau_{-1}\right) e^{-\alpha \tau_{-1}}\right\}
\end{aligned}
$$

is a quasipolynomial in $z=\eta$ for any $\tau$ and $t$, then $(d / d \tau)\left(\tilde{\xi} A(\tau) e^{-\alpha t}\right)$ is a quasipolynomial as well. Hence we have

$$
\left(A^{\prime \prime}(\tau)-\alpha A^{\prime}(\tau)\right)\left\{A\left(\tau_{-2}\right)+A\left(\tau_{-1}\right) e^{\alpha \tau_{-2}}\right\}
$$

is a quasipolynomial in $z$, which is possible only if $A^{\prime \prime}(\tau)-\alpha A^{\prime}(\tau)=0$ or $A\left(\tau_{-2}\right)+A\left(\tau_{-1}\right) e^{\alpha \tau_{-2}}$ is a quasipolynomial in $z$.

In case (a) we have

$$
A^{\prime \prime}(\tau)-\alpha A^{\prime}(\tau)=-\alpha \beta c_{4} \frac{e^{2 \alpha \tau}}{\left(\sqrt{\left(e^{\alpha \tau}-c_{3}\right)^{2}-c_{4}}\right)^{3}}, \quad c_{4}=4 c_{1} c_{2},
$$

and in case (b) we have

$$
A^{\prime \prime}(\tau)-\alpha A^{\prime}(\tau)=-4 c_{1}^{2} \alpha^{-2} e^{2 \alpha \tau}\left(\frac{4 c_{1}}{\alpha^{2}} e^{\alpha \tau}+\frac{c_{2}^{2}-4 c_{1} c_{3}}{\alpha^{2}}\right)^{-3 / 2} .
$$

Therefore, $A^{\prime \prime}(\tau)-\alpha A^{\prime}(\tau)=0$ if $c_{1} c_{2}=0$ in case (a) and if $c_{1}=0$ in case (b). Both these cases are considered in Lemma 3.4.

It follows from $d q / d z=-e^{-\alpha \tau_{-1}}$ that, in case (a), if $r=\sqrt{c_{3}^{2}-4 c_{1} c_{2}} \neq 0$, then

$$
q(\eta)=-\frac{1}{\beta r} \ln \left|\frac{e^{\beta \eta}-p_{1}}{e^{\beta \eta}-p_{2}}\right|, \quad p_{1}=\frac{-c_{3}+r}{2 c_{1}}, \quad p_{2}=\frac{-c_{3}-r}{2 c_{1}},
$$

and if $r=\sqrt{c_{3}^{2}-4 c_{1} c_{2}}=0$, then

$$
q(\eta)=\frac{1}{c_{1} \beta\left(e^{\beta \eta}-p_{1}\right)} .
$$

In case $(b)$, if $r_{1}=\sqrt{c_{2}^{2}-4 c_{1} c_{3}} \neq 0$, then

$$
q(\eta)=-\frac{1}{\beta r_{1}} \ln \left|\frac{\eta-p_{1}^{*}}{\eta-p_{2}^{*}}\right|, \quad p_{1}^{*}=\frac{-c_{2}+r_{1}}{2 c_{1}}, \quad p_{2}^{*}=\frac{-c_{2}-r_{1}}{2 c_{1}},
$$

and if $r_{1}=\sqrt{c_{2}^{2}-4 c_{1} c_{3}}=0$, then

$$
q(\eta)=\frac{1}{c_{1} \beta\left(\eta-p_{1}^{*}\right)}
$$

In case (a) we have 


$$
\begin{aligned}
& \frac{\alpha}{\beta}\left(A\left(\tau_{-2}\right)+A\left(\tau_{-1}\right) e^{\alpha \tau_{-2}}\right)=c_{1} e^{\beta \eta_{-1}}-c_{2} e^{-\beta \eta_{-1}}+\left(c_{1} e^{\beta \eta}-c_{2} e^{-\beta \eta}\right)\left(c_{1} e^{\beta \eta_{-1}}+c_{2} e^{-\beta \eta_{-1}}+c_{3}\right) \\
& =c_{1} e^{\beta \eta_{-1}}\left(c_{1} e^{\beta \eta}-c_{2} e^{-\beta \eta}+1\right)+c_{2} e^{-\beta \eta_{-1}}\left(c_{1} e^{\beta \eta}-c_{2} e^{-\beta \eta}-1\right)+c_{3} c_{1} e^{\beta \eta} \\
& -c_{3} c_{2} e^{-\beta \eta}=c_{1} e^{\beta z_{-1}-\beta q(z)}\left(c_{1} e^{\beta z}-c_{2} e^{-\beta z}+1\right)+c_{2} e^{-\beta z_{-1}+\beta q(z)}\left(c_{1} e^{\beta z}\right. \\
& \left.-c_{2} e^{-\beta z}-1\right)+c_{3} c_{1} e^{\beta z}-c_{3} c_{2} e^{-\beta z} .
\end{aligned}
$$

One can see that $A\left(\tau_{-2}\right)+A\left(\tau_{-1}\right) e^{\alpha \tau_{-2}}$ is a quasipolynomial in case (a) only if $r=\sqrt{c_{3}^{2}-4 c_{1} c_{2}}$ $= \pm 1$. If $r= \pm 1$, function $A\left(t_{1}-t\right)$ becomes $(\beta / \alpha) \sqrt{\left(e^{\alpha\left(t_{1}-t\right)}-c_{3}\right)^{2}-c_{3}^{2}+1}$, where $c_{3}$ is an arbitrary constant, and one of $n$-integrals for $t_{1 x}=t_{x}+(\beta / \alpha) e^{\alpha t} \sqrt{\left(e^{\alpha\left(t_{1}-t\right)}-c_{3}\right)^{2}-c_{3}^{2}+1}$ is $I=(\alpha / 2) t_{x}^{2}-t_{x x}$ $+(\alpha / 2) e^{2 \alpha t}$.

In case (b) direct calculations show that

$$
A\left(\tau_{-2}\right)+A\left(\tau_{-1}\right) e^{\alpha \tau_{-2}}=Q(z)+P\left(z, z_{-1}\right)+J\left(z, z_{-1}\right),
$$

where $Q(z)$ is some function depending only on $z, P\left(z, z_{-1}\right)$ is a polynomial function of two variables, and

$$
J\left(z, z_{-1}\right)=-\frac{2 c_{1}}{\alpha} z_{-1} q(z)\left(2 c_{1} z+c_{2}\right) .
$$

Since $A\left(\tau_{-2}\right)+A\left(\tau_{-1}\right) e^{\alpha \tau_{-2}-P\left(z, z_{-1}\right)=Q(z)+J\left(z, z_{-1}\right)}$ is a quasipolynomial in $z$, then

$$
\frac{\partial\left(Q(z)+J\left(z, z_{-1}\right)\right)}{\partial z_{-1}}=\frac{2 c_{1}}{\alpha} q(z)\left(2 c_{1} z+c_{2}\right)
$$

is also a quasipolynomial in $z$, which is possible only if $r_{1}=\sqrt{c_{2}^{2}-4 c_{1} c_{3}}=0$. If $r_{1}=0$ we have $A\left(t_{1}-t\right)=c e^{(\alpha / 2)\left(t_{1}-t\right)}$, where $c$ is an arbitrary constant, and the corresponding $n$-integral is $I=$ $-(\alpha / 2) t_{x}^{2}+t_{x x}$.

\section{Case 4: $t_{1 x}=t_{x}+c_{4}\left(e^{\alpha t_{1}}-e^{\alpha t}\right)+c_{5}\left(e^{-\alpha t_{1}}-e^{-\alpha t}\right)$}

It is clear that this equation has a nontrivial $n$-integral which is $I=t_{x}-c_{4} e^{\alpha t}+c_{5} e^{-\alpha t}$. It satisfies the equation $D I=I$ since $D I=t_{1 x}-c_{4} e^{\alpha t_{1}}+c_{5} e^{-\alpha t_{1}}=I$.

\section{CONCLUSION}

In this article we studied differential-difference equations of the form (1) from the Darboux integrability point of view. The problem of classification of Darboux integrable chains is studied by reducing it to an adequate algebraic form. We use the fact that the chain (1) is Darboux integrable if and only if its characteristic Lie algebras $L_{x}$ and $L_{n}$ both are of finite dimension to obtain the complete list of Darboux integrable chains of the particular form $t_{1 x}=t_{x}+d\left(t, t_{1}\right)$.

\section{ACKNOWLEDGMENTS}

This work is partially supported by the Scientific and Technological Research Council of Turkey (TÜBİTAK). One of the authors (I.H.) thanks Russian Foundation for Basic Research (RFBR) (Grant Nos. 09-01-92431KE-a, 08-01-00440-a, and 07-01-00081-a).

${ }^{1}$ I. Habibullin, N. Zheltukhina, and A. Pekcan, J. Math. Phys. 49, 102702 (2008).

${ }^{2}$ I. T. Habibullin, Symmetry, Integr. Geom.: Methods Appl. 1, 023 (2005).

${ }^{3}$ I. Habibullin and A. Pekcan, Theor. Math. Phys. 151, 781 (2007).

${ }^{4}$ I. Habibullin, A. Pekcan, and N. Zheltukhina, Turkish Journal of Mathematics 32, 1 (2008).

${ }^{5}$ A. V. Zabrodin, Theor. Math. Phys. 113, 1347 (1997).

${ }^{6}$ F. W. Nijhoff and H. W. Capel, Acta Appl. Math. 39, 133 (1995).

${ }^{7}$ B. Grammaticos, G. Karra, V. Papageorgiou, and A. Ramani, Integrability of Discrete-Time Systems, Chaotic Dynamics, NATO Advanced Studies Institute, Series B: Physics (Plenum, New York, 1992), Vol. 298, pp. 75-90.

${ }^{8}$ I. V. Barashenkov and T. C. van Heerden, Phys. Rev. E 77, 036601 (2008). 
${ }^{9}$ V. E. Adler and S. Ya. Startsev, Theor. Math. Phys. 121, 1484 (1999).

${ }^{10}$ P. S. Laplace, Mémoires de l'Académie Royale des Sciences de Paris 23, 341 (1773) [reprinted in P. S. Laplace, Oevres Complètes (Gauthier-Villars, Paris, 1893), pp. 5-68.

${ }^{11}$ G. Darboux, Leçons sur la Thëorie Gënérale des Surfaces et les Applications Geometriques du Calcul Infinitesimal (Gautier-Villars, Paris, 1915).

${ }^{12}$ I. M. Anderson and N. Kamran, Duke Math. J. 87, 265 (1997).

${ }_{13}^{13}$ A. V. Zhiber and V. V. Sokolov, Russ. Math. Surveys 56, 61 (2001).

${ }^{14}$ A. B. Shabat and R. I. Yamilov, Exponential Systems of Type I and the Cartan Matrices (Bashkirian Branch of Academy of Science of the USSR, Ufa, 1981) (in Russian).

${ }^{15}$ R. K. Dodd and R. K. Bullough, Proc. R. Soc. London, Ser. A 351, 499 (1976). 